\title{
Branching versus Linear Logics Yet Again
}

\author{
José Carmo and Amílcar Sernadas
}

Section of Computer Science, Department of Mathematics, IST, Av. Rovisco Pais, 1096 Lisboa

Codex, Portugal

Keywords: Temporal logics; Linear and branching models; State and path formulas; Frame change operators; Axiomatisations; Completeness; Information systems specification and verification; Safety and liveness

Abstract: A brief overview is given of the temporal logics used in concurrent program verification and in database and systems specification. The properties of the underlying modal frame structures are analysed. The relative advantages of the linear and branching approaches are discussed. The state versus path formulas controversy is revisited. A meta-linear operator $L$ is proposed and compared with the "in all trajectories" operator considered in the language CTL*. The usefulness of the new operator within the context of a layered methodology for database and information systems specification and verification is illustrated. The operator is seen as a "frame change operator" and other interesting operators of this class are referred. Finitary and infinitary axiomatisations are given for the operator $L$. The proof of the completeness of the infinitary axiomatisation is briefly outlined. This proof requires an appropriate extension of the usual Henkin methods.

\section{Introduction}

Temporal logics [Pri67, ReU71] are modal logics [HuC68, HuC84] where the underlying frame structure is seen as a time structure and where the modalities (called temporal or tense operators) reflect this interpretation. Both modal and temporal logics have been used with great success in many fields of Computer Science. Herein we shall be especially interested in their application within the

Correspondence and offprint requests to: José Carmo, Section of Computer Science, Department of Mathematics, IST, Av. Rovisco Pais, 1096 Lisboa Codex, Portugal. 
areas of concurrent programming and database and information systems specification.

Making a brief review, we start by recalling the use of modal logics in the specification of sequential programs (deterministic and nondeterministic). In those logics, a modal necessity operator $\square$ is associated with each program $h$ and it is interpreted through Kripke structures of the form $\left\langle\mathscr{W}, \mathscr{R}^{*}\right\rangle$, where $\mathscr{W}$ represents the set of the computer states and $\omega^{*} \mathscr{R}^{*} \omega^{\prime}$ (that is, $\left.\left\langle\omega, \omega^{\prime}\right\rangle \in \mathscr{R}^{k}\right\rangle$ iff we can obtain the final state $\omega^{\prime}$ executing $h$ from the initial state $w$. The exogenous logics so obtained (using the terminology proposed in [Pnu77]) allow a top-down and compositional construction of the desired program. Particularly important and popular in this context are Dijkstra's predicate transformer [Dij75] and the so called dynamic logics [Pra76, Fil79, Har79] (that concentrate on the class of the regular programs with some extensions to recursive programs).

The previous approach is not appropriate when we consider concurrent programs or programs that should not terminate. In these cases the natural solution consists in associating to each program the set of its possible execution sequences (or paths): the interleaving model (for other more powerful process models capable of representing true concurrency see e.g. [NpW80, Pra87, Rei88]). This view suggests the application of temporal logics for concurrent program specification and verification, as originally proposed in [Pnu77] within the scope of an endogenous logic. Since then, both linear and branching temporal logics have been extensively used in this area (for instance, [Pnu79, Gab80, MaP81, MaP83] use linear logics and [BMP81, ClE81, EmH82] use branching logics) and a great controversy has emerged on which approach best fulfils the purpose at hand. Remarkable papers on this discussion are [Lam80, EmH86]. Finally, it should be stressed that formalisms have also been proposed incorporating temporal operators into dynamic logic (see e.g. [Nis80, HKP82]).

In the database and information system area [Bub77, RLR79] recognised the need for expressing requirements involving time and [Ser80] proposed the adoption of temporal formalisms for that purpose. Since then, temporal formalisms have been widely used (see e.g. [CCF82, Kun84, Car85, LEG85, VeF85, FiS86, FuN86]), as well as some other modal logics (see e.g. [GMS83, MKJ86]). Although most works in this area adopt linear formalisms (see e.g. [CaF82, Kun84, LEG85, VeF85, FiS86, FuN86, FiS88]), others [CaS88a, CaS88b] show the advantages of also considering branching formalisms in database specification/verification. Thus, the controversy between linear and branching logics becomes relevant also in the database and information system area and one of the topics of this paper consists in the re-examination of that controversy taking into account the specific issues of this area.

Research on database dynamics is relatively recent and usually (see e.g. [Ser80, CCF82, Kun84, FuN86]) is concerned almost exclusively with the description of safety requirements on the database and with the definition of the update operations: the former describe the possible database states (static constraints) and sequences of database states (transition constraints) and are usually expressed using many-sorted temporal languages; the latter describe the update operations by their properties using event abstractions and pre and post conditions.

Recently, the Infolog approach to systems specification [SeS83, SeS85] stressed the need for considering both safety and liveness aspects when specifying reactive dynamic information systems, taking into account the tools introduced in the programming area [Pnu77, MaP81, OwL82] for specifying the liveness 
requirements (see e.g. [Car85, FiS86, CaS88a, FiS88]). Briefly, the Infolog approach views an information system as an independent agent that keeps its own database, can communicate with other systems through messages and is composed of concurrent subsystems sharing the database. This view stresses that when we want to specify such systems we must specify not only the possible database states and the effects of the operations on those states, but also the reaction patterns of the system, its processes and its communication behaviour. Hence, this approach originally established a bridge between the area of logical specification of databases and the area of logical specification of concurrent processes.

It should be stressed that in the Infolog approach no attempt is made to associate each database component with the process corresponding to its displayed behaviour. Such an integration of the database structure with the activity structure (within each information system) leads to an object-oriented approach [SSE87, ESS88, SFS88, SEC89], where the same concept should be used to model both the static components (e.g. "records") and the dynamic components ("processes"), and it is an important field of research nowadays.

Finally, in order to handle such complex entities like the envisaged information systems, layered methodologies have been proposed since the pioneering work reported in [CCF82]. In [CaS88a, CaS88b], the layered methodology of [CCF82, FuN86] is extended in order to include the specification of the system behaviour (that is, the activities that the system is required to perform plus their triggers), as well as the specification of its communication behaviour. Note that although data integrity constraints are usually considered simply as safety constraints in the programming theory literature, as well as within the general context of the reactive systems specification and development methodologies (see e.g. [Pnu86]), their importance in the database area justifies a separated treatment in a specific layer, leaving the update operations to be described in a subsequent layer in such a way that no data integrity constraint is violated (as in [CCF82, FuN86]). In [CaS88a, CaS88b] the same strategy is adopted, but further layers are introduced in order to specify the liveness and other requirements on the system evolution and communication, as well as to define the processes that should enforce them.

In order to support the proposed layered methodology, a unifying logic framework is introduced therein (using purely semantic techniques in [CaS88a] and both semantic and axiomatic techniques in [CaS88b]). The framework includes linear logics for some layers and branching logics for other layers, depending on the type of requirements and constraints relevant in each of them. In order to establish the "compliance relation" that should exist between each layer specification and the previous layer specification, a "for all trajectories" operator seems to be necessary. The meta-linear operator $L$ proposed in [Car85] was chosen, since, as we shall discuss later on, it seems more appropriate for the purposes at hand than the operator "in all trajectories" considered in the language CTL* [EmH86].

The semantics and an axiomatisation of the operator $L$ are given in this paper. The operator allows the combination of a pure branching logic (where no reference to the trajectories is explicited considered) with the underlying linear logic, in such a way that the branching logic so obtained incorporates the latter through that operator. Moreover, the same results can be obtained axiomatically, by choosing appropriate axioms and rules for the operator $L$ and correctly combining the axiomatisation for the pure branching logic with its extension that axiomatises 
the underlying linear logic. Note that we use throughout this paper the term logic to refer to a formal language and a truth semantics (based on the naive set theory) that associates a precise meaning to that language.

The basic idea in the semantic definition of the operator $L$ is to change the frame of the semantic analysis from the branching structure to the underlying linear structure. This idea seems to be fruitfull and other examples of what we will call frame change operators will be briefiy discussed.

Both finitary and infinitary sound axiomatisations are given for the operator $L$, in a propositional setting. Both axiomatisations are based in the same finitary language, but the infinitary axiomatisation includes a set of infinitary inference rules (besides some finitary inference rules and some, obviously finitary, axiom schemas). The proof of the completeness of the infinitary axiomatisation is briefly outlined herein. This proof originally extends the usual Henkin constructions based on the "maximal consistent sets" by an appropriate definition of the concept of world that is necessary for the construction at hand, in virtue of the frame change character of $L$. All the details of this construction, as well as the proofs omitted herein, can be found in [Car88, CaS89b].

A short description of each section now follows. Section 2 includes an overview of the main temporal formalisms used in concurrent program verification and in database and systems specification, briefly discusses the properties of the underlying time structure (frame) considered in the two areas, revisits the branching versus path formulas controversy and compares the "for all trajectories" operator proposed in [Car85] (also called herein the meta-linear operator) and the one considered in the language CTL*. Section 3 sketches the layered methodology to systems specification described in [CaS88a, CaS88b]. Section 4 compares the linear and branching approaches, in general and within the context of the specific layered methodology. Finitary and infinitary sound axiomatisations for the operator $L$ are presented in Section 5 and a brief outline of the proof of the completeness of the infinitary axiomatisation is given in Section 6. Finally, conclusions and some directions for further work are included in Section 7.

\section{Temporal Formalisms}

\subsection{Preliminaries}

A temporal language $\mathscr{L}$ is a propositional or first-order (many-sorted or not) language $\mathscr{L}_{0}$ plus a set of temporal operators.

The semantics of a temporal language $\mathscr{L}$ is defined as follows. A frame [HuC84] (time structure, or world structure) $\mathscr{F}$, for $\mathscr{L}$, is usually defined in the philosophic and formal logic area as a Kripke structure of the form $(\mathscr{W}, \mathscr{R})$, where $\mathscr{W}$ is a nonempty set, the set of worlds, and $\mathscr{R}$ is a binary relation over $\mathscr{W}$, satisfying some requirements, called the accessibility relation (see e.g. [ReU71, McA76, HuC84]). An interpretation structure (called model in e.g. [HuC84]) $\mathscr{F}$ for $\mathscr{L}$ is a structure of the form $(\mathscr{F}, \mathcal{M})$ where $\mathscr{M}$ maps each world $\omega$ (in $\mathscr{F}$ ) into an interpretation structure for the associated nontemporal language $\mathscr{L}_{0}$ (we usually write $\mathscr{I}=(\mathscr{W}, \mathscr{R}, \mathscr{M})$, instead of $\mathscr{I}=(\mathscr{F}, \mathscr{M})$ ). Finally, the basic semantic unit is the situation. By situation (historical moment in [McA76]) we mean a structure consisting of: an interpretation structure $\mathscr{F}$; a world $\omega$ in $\mathscr{I}$, that denotes the present (or current) world; possibly some other temporal elements obtained 
from $\mathscr{I}$ (usually none); and a valuation of the variables in the different worlds, if $\mathscr{L}_{0}$ is first-order.

Using the previous structures, we define the semantic notions of validity, truth, satisfaction and entailment as follows. A formula $f$ is valid, written $\vDash f$, iff it is true in all frames. A formula $f$ is true in a frame $\mathscr{F}$, written $\mathscr{F} F f$, iff $f$ is true in all interpretation structures on $\mathscr{F}$. A formula $f$ is true in an interpretation structure $\mathscr{I}$, written $\mathscr{I} \vDash f$, iff $f$ is satisfied by all situations on $\mathscr{I}$. The satisfaction of a formula $f$ by a situation sit, written sit $\models f$, is defined as usual if no temporal operator is the main operator in $f$. For defining the satisfaction of the formulas constructed with temporal operators we use the time structure and the other temporal elements in sit, as we shall see examples later. Finally, we say that a set of formulas $A$ semantically entails a formula $f$, written $A \models f$, iff $f$ is true in every interpretation structure where all formulas in $A$ are true (obviously, $\varnothing \vDash f$ iff $\vDash f)$. For a discussion of other possible notions of semantic entailment see [CaS89a].

It should be stressed that we are using the term temporal logic to refer to the classical temporal logics where the basic time unit is the time instant (or world), and not a period of time, and $\mathscr{R}$ means "earlier than" (about logics of time intervals see instead e.g. [Ben82, All83, Sch85, Ven88]). Moreover, herein we will not consider temporal languages including recursion (some references to those logics can be found in [Sti87]).

In order to simplify the presentation, throughout the rest of this section we shall assume that $\mathscr{L}$ is a propositional temporal language. The propositional symbols will be generically denoted by $p, p^{\prime}, \ldots$ and the primitive propositional conectives will be $\neg$ (not) and $\Rightarrow$ (implies). The other propositional conectives: $\wedge$ (and), $\vee$ (or) and $\Leftrightarrow$ (equivalent) are defined as usually by abbreviation rules. Thus, in the sequel, $\mathcal{M}(\mathscr{\omega})(p) \in\{0,1\}$ for every interpretation structure $\mathscr{I}$ of $\mathscr{L}$. Moreover:

$$
\begin{array}{ll}
(\mathscr{I}, \omega, \ldots) \vDash p & \text { iff } \mathcal{M}(w)(p)=1 \\
(\mathscr{I}, w, \ldots) \vDash(\neg f) & \text { iff not }(\mathscr{I}, \omega, \ldots) \vDash f \\
(\mathscr{I}, w, \ldots) \vDash\left(f_{1} \Rightarrow f_{2}\right) & \text { iff not }(\mathscr{I}, w, \ldots) \vDash f_{1} \text { or }(\mathscr{I}, \omega, \ldots) \vDash f_{2}
\end{array}
$$

Some notation follows: the metasymbols $w^{2}, w_{1}, w^{\prime}, \ldots$ denote generic elements of $\mathscr{W}$, and $f, \ldots$ denote generic formulas of $\mathscr{L} ; F: A \rightarrow B$ means that $F$ is a total mapping between $A$ and $B$, and $F \uparrow C$ denotes the restriction of $F$ to the subset $C$ of $A$; if $\mathscr{R}$ is a binary relation over $\mathscr{W}$, we write $w \mathscr{R} w^{\prime}$ instead of $\left\langle\omega, w^{\prime}\right\rangle \in \mathscr{R}$, $\mathscr{R} \uparrow C$ denotes the restriction of $\mathscr{R}$ to the subset $C$ of $\mathscr{W}$ (that is, $\mathscr{R} \uparrow C=\mathscr{R} \cap$ $(C \otimes C)$, where $\otimes$ denotes the cartesian product), $\omega \mathscr{R} \omega_{1} \mathscr{R} \omega_{2}$ is an abbreviation of $\omega_{R} \omega_{1}$ and $\omega_{1} \mathscr{R} \omega_{2}$, and $S^{\mathscr{N}}$ is the binary relation over $\mathscr{W}$ so defined: $\omega^{\mathscr{R}^{2}} \omega_{1}$ iff $\omega_{R} \omega_{1}$ and for no $\omega_{2}$ we have $\omega_{R} \omega_{2} \mathscr{R} \omega_{1}\left(S^{\mathscr{H}}\right.$ is the "step" relation of $\left.\mathscr{R}\right)$. Furthermore, we sometimes also use the symbols $\neg, \Rightarrow, \wedge, \vee, \Leftrightarrow, \forall$ and $\exists$ as symbols of the metalanguage, in order to shorten the relevant meta-expressions, even when discussing interpretations. With respect to the syntax, parentheses will be omitted whenever the meaning of the resulting "formulae" is perfectly clear (unary operators have higher priority).

When defining temporal logics, there are two possible views with respect to the underlying nature of future time. One is that time is linear: at each moment there is only one possible future. The other is that time has a branching, tree-like nature: at each moment there may be different possible futures. We shall consider 
both views. On the other hand, we adopt throughout this paper the linear view of the past.

\subsection{Linear Formalisms}

In this subsection we are going to discuss the properties of the underlying time structure and the temporal operators usually considered in the linear formalisms.

In the philosophic and formal logic area, when considering linear formalisms, the situations have the form $(\mathscr{I}, w)$ and the time structure $\mathscr{F}=(\mathscr{W}, \mathscr{R})$ in $\mathscr{I}$ should satisfy at least the following conditions (see e.g. [ReU71, McA76]): $\mathscr{R}$ is transitive and left and right linear over $\mathscr{W}$ (for instance, the left linearity of $\mathscr{R}$ means that $\left.\left(\omega_{2} \mathscr{R} \omega_{1} \wedge \omega_{3} \mathscr{R} \omega_{1}\right) \Rightarrow\left(\omega_{2} \mathscr{R} \omega_{3} \vee \omega_{3} \mathscr{R} \omega_{2} \vee \omega_{2}=\omega_{3}\right)\right)$.

In the programming area, when considering linear formalisms, the time structure is a finite, or infinite, sequence of program states $\left\langle\diamond_{0}, \sigma_{1}, \ldots\right\rangle$ that corresponds to a possible execution of the program starting with the initial state $\triangle_{0}$ (see e.g. [Pnu79, MaP81]). On the other hand, as discussed e.g. in [CaS88a], in the database area the time structure is a possible sequence of database states $\left\langle s_{0}, \diamond_{1}, \ldots\right\rangle$, where $\diamond_{0}$ is the initial database state. These time structures can be included in the class of the Kripke structures, as defined above, by considering $\mathscr{W}$ as the set of all (finite) prefixes of the above sequence and $\mathscr{R}$ as the relation: $\omega \mathscr{R} \omega^{\prime}$ iff $\omega$ is a proper prefix of $\omega^{\prime}$. Although the above time structures can be incorporated into the class of the Kripke structures in other ways (e.g. by identifying each world with a program or database state and $\mathscr{R}$ with the precedence relation between states), the chosen solution seems to be the most adequate for expressing the characteristics of those time structures (namely avoiding a circular accessibility relation). Thus, we shall be particularly interested in time structures $(\mathscr{W}, \mathscr{R})$ where $\mathscr{R}$ is a strict linear order $(<)$ over $\mathscr{W}$ (that is, $\mathscr{R}$ is irreflexive and transitive, and any two distinct elements of $\mathscr{W}$ are comparable by $\mathscr{R}$ ) such that $\left\{w^{\prime}: w^{\prime} \mathscr{R} w\right\}$ is finite for every $w$ in $\mathscr{W}$.

The main temporal operators usually considered in the linear formalisms are: $G$ (always in the future), $G_{0}$ (in the present and always in the future), $H$ (always in the past), $H_{0}$ (in the present and always in the past), the "until" operators $U$ and $U_{0}$, the "since" operators $S$ and $S_{0}$, and when the time structure is discrete, like the chain above, the operators $X$ (in the next world) and $Y$ (in the previous world). It should be stressed that although in the philosophic and formal logic area both future and past operators are usually considered, in the programming area, with few exceptions (see e.g. [LPZ85, Pnu86]), only future operators are considered. In the database area both approaches have been taken: for instance, [CaF82, LEG85, Lip88] only consider future operators and [CCF82, Car85, Kun85, FiS86] consider both future and past operators.

We will identify each kind of temporal formalism by specifying if it is linear or branching and by identifying the (primitive) temporal operators it uses. Of course, we may also obtain different logics by choosing a different nontemporal component for the language (propositional or not, and so on), or by imposing different properties on the time structure as, for instance, a last time instant. Note, however, that the latter choice only leads to different axiomatisations if the relevant properties are expressible using the chosen temporal operators: for instance, the irreflexivity of $\mathscr{R}$ is not expressible only using temporal operators [ReU71, McA76], although we can express irreflexivity in combination with other properties, like the chain above [Boo79]. It should be stressed that some of the 
works mentioned below use different symbols from the ones adopted herein to identify the same temporal operators.

The precise meaning of the operators above is defined as follows (where $(\mathscr{F}, \infty)$ is the general form of a situation in this linear formalism):

$$
\begin{aligned}
& (\mathscr{Y}, w) \vDash(G f) \quad \text { iff }\left(\forall w^{\prime}\right)\left(w \mathscr{R} w^{\prime} \Rightarrow\left(\mathscr{F}, w^{\prime}\right) \vDash f\right) \\
& (\mathscr{I}, \omega) \vDash\left(G_{0 f}\right) \quad \text { iff }\left(\forall \omega^{\prime}\right)\left(\left(\omega \mathscr{R} \omega^{\prime} \vee \omega=\omega^{\prime}\right) \Rightarrow\left(\mathscr{I}, \omega^{\prime}\right) \vDash f\right) \\
& (\mathscr{F}, \omega) \vDash(H \ell) \quad \text { iff }\left(\forall \omega^{\prime}\right)\left(\omega^{\prime} \mathscr{R} \omega \Rightarrow\left(\mathscr{F}, w^{\prime}\right) \vDash f\right) \\
& (\mathscr{I}, \omega) \vDash\left(H_{0} f\right) \quad \text { iff }\left(\forall \omega^{\prime}\right)\left(\left(\omega^{\prime} \mathscr{R} \omega \vee \omega=\omega^{\prime}\right) \Rightarrow\left(\mathscr{I}, \omega^{\prime}\right) \vDash f\right) \\
& (\Phi, \omega) \equiv(X f) \quad \text { iff }\left(\exists \omega^{\prime}\right)\left(\omega S^{\mathscr{T}} \omega^{\prime} \wedge\left(\mathscr{I}, \omega^{\prime}\right) \vDash f\right) \\
& (\mathscr{I}, \omega) \vDash(Y f) \quad \text { iff }\left(\exists \omega^{\prime}\right)\left(\omega^{\prime} S^{s \phi} \omega \wedge\left(\mathscr{J}, \omega^{\prime}\right) \models f\right) \\
& (\mathscr{I}, \omega) \vDash\left(f_{1} \cup f_{2}\right) \quad \text { iff }\left(\exists \omega_{2}\right)\left(\omega \mathscr{R} \omega_{2} \wedge\left(\mathscr{I}_{,}, \omega_{2}\right) \vDash f_{2} \wedge\right. \\
& \left.\left(\forall w_{1}\right)\left(\omega_{R} \mathscr{R} w_{1} \mathscr{R} w_{2} \Rightarrow\left(\mathscr{G}, w_{1}\right) \neq f_{1}\right)\right) \\
& (\mathscr{I}, \omega) \equiv\left(f_{1} U_{0} f_{2}\right) \quad \text { iff }(\mathscr{I}, \omega) \vDash f_{2} \vee\left(\exists \omega_{2}\right)\left(\omega \mathscr{R} \omega_{2} \wedge\left(\mathscr{I}, \omega_{2}\right) \vDash f_{2} \wedge\right. \\
& \left.\left(\forall \omega_{1}\right)\left(\left(\omega_{1}=\omega \vee \omega \mathscr{R} \omega_{1} \mathscr{R} \omega_{2}\right) \Rightarrow\left(\mathscr{T}, \omega_{1}\right) \vDash f_{1}\right)\right) \\
& \left(\mathscr{\Phi}, \omega^{\prime}\right)=\left(f_{1} S f_{2}\right) \quad \text { iff }\left(\exists w_{2}\right)\left(w_{2} R w_{\wedge}\left(\mathscr{I}, w_{2}\right) \vDash f_{2} \wedge\right. \\
& \left.\left(\forall \omega_{1}\right)\left(\omega_{2} R \omega_{1} \mathscr{R} \omega\left(\mathscr{I}_{,}, \omega_{1}\right) \vDash A_{1}\right)\right) \\
& (\mathscr{F}, w) \vDash\left(f_{1} S_{0} f 2\right) \quad \text { iff }(\mathscr{I}, \omega) \vDash f_{2} \vee\left(\exists \omega_{2}\right)\left(\omega_{2} \mathscr{R} \omega \wedge\left(\mathscr{I}, \omega_{2}\right) \vDash f_{2} \wedge\right. \\
& \left.\left(\forall \omega_{1}\right)\left(\left(\omega_{1}=\omega \vee \omega_{2} \mathscr{R} \omega_{1} \mathscr{R} \omega\right) \Rightarrow\left(\mathscr{S}_{,} \omega_{1}\right) \vDash f_{1}\right)\right)
\end{aligned}
$$

It should be noted that in the works of the areas of programming and databases, where only future operators are considered, the semantics of those operators is usually defined in a different way. For instance, instead of defining the semantics of the $G$ operator as: $\left(\left(\Delta_{0}, \Delta_{1}, \ldots\right\rangle, s_{i}\right) \models(G f)$ iff $(\forall r>i)\left(\left(\Delta_{0}, \Delta_{1}, \ldots\right\rangle, \Delta_{r}\right) \vDash f$, similarly to what is done above (and forgetting the component $M$ of $\mathscr{I}$ ), they usually define it as follows: $\left(\delta_{i}, \Delta_{i+1}, \ldots\right\rangle \models(G f)$ iff $(\forall r>i)\left(\Delta_{r}, \Delta_{r+1}, \ldots\right) \neq f$. Although the two semantics are equivalent, contrarily to what happens with the semantics above, where the state is the basic semantic unit, the latter semantics takes the path as the basic semantic unit, the satisfaction is defined over a path, and the time structure is a set of paths consisting of an initial path and all its proper suffixes (more generally, we may consider as a time structure any set of paths that must obviously satisfy the suffix closure property [Lam80, Eme83]). In both semantics, the meaning of the nontemporal component of the language is state dependent.

Many other (nonprimitive) temporal operators can be defined. For instance, $F, F_{0}, P$ and $P_{0}$ will be used throughout this paper to denote the duals of $G$, $G_{0}, H$ and $H_{0}$ (that is, e.g. $F$ is $\neg G \neg$ ). We also use $N$ (resp. $B$ ) to denote the dual of $X$ (resp. $Y$ ), although they are equivalent whenever we only consider nonnegative integer time (resp. integer time) structures. The "precede" operators: $\operatorname{Pr}$ and $\mathrm{Pr}_{0}$, useful for expressing safety properties [MaP83], are defined as follows: $\left(f_{1} \operatorname{Pr} f_{2}\right)$ is $\neg\left(\left(\neg f_{1}\right) \cup f_{2}\right)$ and $\left(f_{1} \operatorname{Pr}_{0} f_{2}\right)$ is $\neg\left(\left(\neg f_{1}\right) U_{0} f_{2}\right)$. The "always $f_{1}$ until $f_{2}$ " operator considered in [LEG85, Lip88] is defined as $\left(\left(f_{1} U_{0} f_{2}\right) \mathrm{V}\right.$ $\left.\left(G_{0} f_{1}\right)\right)$, and reciprocally we define $\left(f_{1} U_{0} f_{2}\right)$ as $\left(\left(F_{0} f_{2}\right) \wedge\left(\right.\right.$ always $f_{1}$ until $\left.\left.f_{2}\right)\right)$. 
The operators $G_{0}, H_{0}, U_{0}$ and $S_{0}$ are defined in terms, respectively, of $G, H, U$ and $S$, but the converse is not true. For instance, $\left(f_{1} U_{0} f_{2}\right)$ is defined as $\left(\left(\left(f_{1} U f_{2}\right) \wedge\right.\right.$ $\left.\left.f_{1}\right) \vee f_{2}\right)$ ). The operators $G$ and $X$ can be defined in terms of $U$, as follows [GPS80]: $(G f)$ is $\neg((f \vee \neg f) U \neg f)$ and $(X f)$ is $((f \wedge \neg f) U f)$, but the converse is not true. Analogous comments can be made about the correspondent past operators. Moreover, in [Kam68] it is proved that the operators $U$ and $S$ are descriptively complete on the class of interpretation structures where $\mathscr{R}$ is a complete linear order over $\mathscr{W}$, that is, any temporal operator can be defined using these operators when we only consider such time structures. In [GPS80] it is also proved that the operator $U$ alone plays the same role when we only consider future temporal operators.

In short, we can classify: the axiomatic system $K_{l}$ in [ReU71] (called CL in [McA76]) and the temporal logics in [Kun84, Kun85] as belonging to the formalism linear $(G, H)$; the temporal logic in [LEG85] as linear $\left(G_{0}, U_{0}\right)$ (that is, more precisely, linear $\left.\left(U_{0}\right)\right)$; the temporal logics in [FiS86, FiS88] as linear $(G, H, X, Y)$ (although therein we also can define the operators $X$ and $Y$, as well as the operators $U$ and $S$, as nonprimitives, using the event-occurrence predicate common to all Infolog logics); the temporal logic in [OwL82] and the linear logic referred to in [Lam80] as linear $\left(G_{0}\right)$; the axiomatic system $D X$ defined in [Pnu79] as linear $\left(G_{0}, X\right)$; the temporal logics in [MaP81, MaP83] and the one in [Lip88] as linear $\left(X, U_{0}\right)$; the axiomatic systems $D X$ and $D U X$ defined in [GPS80] as, respectively, linear $(G, X)$ and linear $(U)$; and the temporal logics in [LPZ85, Pnu86] as linear $\left(X, Y, U_{0}, S_{0}\right)$.

\subsection{Branching Formalisms}

Assuming that the past is linear, the minimal conditions we should impose on the time structure $(\mathscr{W}, \mathscr{R})$ underlying a branching logic are those imposed in $[\operatorname{ReU} 71, \mathrm{McA} 76]$ with respect to the branching system $K_{\ell}$, namely that $\mathscr{R}$ should be transitive and left linear over $\mathscr{W}$. Moreover, when we adopt the branching view we usually need to consider some temporal operators (not considered in $K_{b}$ ) that reflect the branching nature of time by allowing quantification over the different possible futures in their semantic definition. Otherwise, the expressibility power of such logics will be inadequate for practical purposes at hand. Note that if we define $F f$ as in the previous subsection, then $F f$ will only mean "possibly $f$ ", instead of "eventually $f$ ", when $(\mathscr{W}, \mathscr{R})$ is not linear.

Hence, for defining branching logics it is first necessary to describe which are the possible trajectories. This is achieved in the philosophic and formal logic area by identifying the trajectories with the maximal $\mathscr{R}$-chains on $\mathscr{W}$ (see e.g. [McA76, McC79]). On the other hand, with respect to the semantics of the branching operators there are two alternative approaches - the Peircean and the Ockhamist [Pri67] (Antactualist and Actualist in [Bur78]). Although these two schools of thought have appeared in the philosophic and formal logic area motivated by different philosophical views of handling nondeterministic time, issues not discussed herein (see instead e.g. [Pri67, Tho70, McA76, Bur78]), we can distinguish the two schools by the types of situations they consider. This leads to a similar classification of the branching logics considered in the programming area (in the database area only the Peircean approach has been considered), although therein the motivation for the two semantic approaches is completely different, namely pragmatic. However, before characterising the two approaches, 
we are going to briefly analyse the time structures considered in the programming and database areas.

In the programming area, when considering branching formalisms, each time structure is identified with a computation tree of the program, that is, a set of alternative paths for the program execution starting in the same initial state (if we only are interested in future operators, then the last condition is usually omitted and a time structure is identified with a set of paths having the suffix closure property [Lam80]). Other conditions can be imposed on the set of alternative paths in order to guarantee that the abstract semantics reflects the essential properties of the "real" concurrent programs. For instance, in order to guarantee that a program's future behaviour only depends on its current state, it is sometimes required that the set of alternative paths possesses the fusion closure property: if a program state is common to two paths then the concatenation of the prefix of any one of them with the suffix of the other, respectively, ending and starting in that common state is still a path (see e.g. [Eme83, EmH86]).

As for the linear formalisms, we include the previous branching time structure in the class of the Kripke structures by considering $\mathscr{W}$ as the set of all (finite) prefixes of the alternative paths and $\mathscr{R}$ as the relation: $w \mathscr{R} \omega$ iff $w$ is a proper prefix of $\omega^{\prime}$. However, in this way the set of paths may not correspond to the set of all the maximal $\mathscr{R}$-chains on $\mathcal{W}$, although each path can obviously be identified with a maximal $\mathscr{R}$-chain on $\mathscr{W}$ (the set of its prefixes). In order to guarantee that correspondence it is necessary that the set of alternative paths satisfy in addition the limit closure property, that is [Eme83]: whenever each of all the (finite) prefixes of an infinite sequence of states belongs to some path in the set, that sequence also belongs to the set of alternative paths. Since this limit closure constraint can be violated when discussing programs executing under fair scheduling (see e.g. [Eme83]), we prefer to not impose such a constraint (the other option is supported in [Eme83]). Thus, in order to include these time structures in the class of the Kripke structures, we must extend these with another component $\mathscr{T}$ (a bundle according to the terminology in [Bur78]) that denotes the set of all (considered possible) trajectories and that satisfies the following properties: (1) each $t$ in $\mathscr{T}$ is a maximal $\mathscr{R}$-chain on $\mathscr{W} ;(2)$ for each $w$ in $\mathscr{W}$ there is a trajectory $t$ (in $\mathscr{T}$ ) that passes through $w$, that is, such that $w \in t$ (this second condition corresponds to the state completeness property described in [EmH86]). Using the terminology of [Sti87], we say that such an interpretation structure $\mathscr{I}=(\mathscr{W}, \mathscr{R}, \mathscr{T}, \mathscr{M})$ is saturated whenever $\mathscr{T}$ coincides with the set of all maximal $\mathscr{R}$-chains on $\mathscr{W}$.

In the database area, the semantic role of the program execution paths is performed by the different possible sequences of database states, or by the sequences of events (if we identify, like in the Infolog model, each atomic transition of database state with an occurrence of a distinct event), and so most of the previous discussion applies herein. However, it should be noted that some of the conditions imposed on each set of alternative paths in the programming area may be meaningless in the database area. Namely, we consider inadequate to impose the fusion closure property in this area since we support that, at the conceptual level, it is not necessary that all the history of the database is recorded in its current valuation: this allows specifications with high degrees of memory independence [Ser80] where the decision on what to keep in the current valuation from previous valuations can be delayed to later stages of the design and implementation. In summary, we should consider interpretation structures $\mathscr{I}=$ $(\mathscr{W}, \mathscr{R}, \mathscr{T}, \mathcal{M})$, where $\mathscr{W}, \mathscr{T}$ and $\mathscr{M}$ are as before, and $\mathscr{R}$ is irreflexive, transitive, 
left linear, has a minimal element $\omega_{0}$ (that is, if $\omega_{\neq} \omega_{0}$ then $\omega_{0} \mathscr{R} \omega$ ) and for every $w$ in $\mathscr{W}$ the set $\left\{w^{\prime}: w^{\prime} \mathscr{R} \omega\right\}$ is finite.

On the other hand, in the database area we may also want to accept (as in e.g. [Car85]) that after some event occurrence any of the two following situations may happen: (a) other events do occur; or (b) nothing more happens in the system's life. For this purpose, we must change the condition 1 in the above definition of $\mathscr{T}$ to: $\left(1^{\prime}\right)$ each $t$ in $\mathscr{T}$ is a nonempty subset of $\mathscr{W}$ such that any two distinct worlds in $t$ are comparable through $\mathscr{R}$ and whenever we have $\mathscr{R}^{\prime} w^{\prime}$ for $w \in \mathscr{W}$ and $w^{\prime} \in t$, $w$ must also belong to $t$ (we shall call the subsets of $\mathscr{W}$ that fulfil this condition left maximal $\mathscr{R}$-chains on $\mathscr{W})$. Note that for the relations $\mathscr{R}$ in which we are interested (described above) this condition $1^{\prime}$ is equivalent to state that any $t$ in $\mathscr{T}$ is of the form ( $\left.\left\{\omega^{\prime}: \omega^{\prime} \mathscr{R} \omega\right\} \cup\{\omega\}\right)$ for some $\omega$ in $\mathscr{W}$. We shall call possibly terminal worlds the worlds $w$ that are terminal according to some trajectory in $\mathscr{T}$ (that is, such that $\left(\forall \omega^{\prime}\right) \neg\left(w \mathscr{R} \uparrow t w^{\prime}\right)$ for some $t$ in $\mathscr{T}$ ), and terminal worlds the worlds $w$ that satisfy the condition $\left(\forall w^{\prime}\right) \neg(w \mathscr{R} w)$. Denoting by $\mathscr{W}_{P T}$ and $\mathscr{W}_{T}$ the two previous set of worlds, we obviously have that $\mathscr{W}_{T} \subset \mathscr{W}_{P T}$. In the interpretation structures considered above for the programming applications we have that $\mathscr{W}_{T}=\mathscr{W}_{P T}$.

The Peircean approach appears chronologically first in the programming area and it is based on the situations of the form $(\mathscr{I}, \omega)$, where $w$ denotes the present (or current) world. The primitive temporal operators usually considered within this approach are defined as follows (where $t$ denotes a generic element of $\mathscr{T}$ ):

$$
\begin{aligned}
& (\mathscr{I}, \omega) \vDash(G f) \quad \text { iff }\left(\forall \omega^{\prime}\right)\left(\omega \mathscr{R} \omega^{\prime} \Rightarrow\left(\mathscr{I}, \omega^{\prime}\right) \vDash f\right) \\
& (\mathscr{I}, \omega) \vDash(H f) \quad \text { iff }\left(\forall w^{\prime}\right)\left(w \mathscr{R} w \Rightarrow\left(\mathscr{I}, w^{\prime}\right) \vDash f\right) \\
& (\mathscr{I}, \omega) \vDash(X f) \quad \text { iff }\left(\exists \omega^{\prime}\right)\left(\omega S^{\mathscr{N}} \omega^{\prime} \wedge\left(\mathscr{I}^{\mathscr{f}}, \omega^{\prime}\right) \vDash f\right) \\
& (\mathscr{I}, \omega) \vDash(Y f) \quad \text { iff }\left(\exists \omega^{\prime}\right)\left(w^{\prime} S^{\mathscr{A}} \omega \wedge\left(\Phi^{\prime}, \omega^{\prime}\right) \vDash f\right) \\
& (\mathscr{I}, w) \models\left(F^{+} f\right) \quad \text { iff }(\forall t)\left(\omega \in t \Rightarrow\left(\exists w^{\prime}\right)\left(w \mathscr{R} \omega^{\prime} \wedge w^{\prime} \in t \wedge\left(\mathscr{I}, w^{\prime}\right) \models f\right)\right) \\
& (\mathscr{I}, \omega) \vDash\left(f_{1} U f_{2}\right) \quad \text { iff }(\forall t)\left(\omega \in t \Rightarrow ( \exists \omega _ { 2 } ) \left(\omega \mathscr{R} \omega_{2} \wedge \omega_{2} \in t \wedge\left(\mathscr{I}, \omega_{2}\right) \vDash f_{2} \wedge\right.\right. \\
& \left.\left.\left(\forall \omega_{1}\right)\left(w \mathscr{R} \omega_{1} \mathscr{R} \omega_{2} \Rightarrow\left(\mathscr{I}, w_{1}\right) \vDash f_{1}\right)\right)\right) \\
& (\mathscr{I}, w)=\left(f_{1} S f_{2}\right) \quad \text { iff }\left(\exists w_{2}\right)\left(w_{2} \mathscr{R} w \wedge\left(\mathscr{I}, w_{2}\right) \models f_{2} \wedge\right. \\
& \left.\left(\forall w_{1}\right)\left(w_{2} \mathscr{R} w_{1} \mathscr{R} \omega \Rightarrow\left(\mathscr{I}, w_{1}\right) \vDash f_{1}\right)\right) \\
& (\Phi, \omega) \vDash\left(f_{1} \operatorname{Pr} f_{2}\right) \quad \text { iff }(\forall t)\left(\omega \in t \Rightarrow ( \forall \omega _ { 2 } ) \left(\left(\omega \mathscr{R} \omega_{2} \wedge \omega_{2} \in t \wedge\right.\right.\right. \\
& \left.\left.\left.\left(\mathscr{I}, \omega_{2}\right) \vDash f_{2}\right) \Rightarrow\left(\exists \omega_{1}\right)\left(\omega_{R} \mathscr{R} \omega_{1} \mathscr{R} \omega_{2} \wedge\left(\mathscr{I}, \omega_{1}\right) \vDash f_{1}\right)\right)\right)
\end{aligned}
$$

We can also define temporal operators $G_{0}, H_{0}, F_{0}^{+}, U_{0}, S_{0}$ and $P_{0}$ in a similar way, but taking also into account the present world (like in the linear formalisms). $F, P, N, B$ and $G^{-}$will denote the duals of $G, H, X, Y$ and $F^{+}$. Although we use the same symbols, the meaning of the operators $F$ and $X$ is different from the one given to them in the linear formalisms (here $F f$ informally means "in some possible future world $f$ holds" and $X f$ means that "there exists some next world where $f$ holds"). Contrary to what happens in the linear formalisms, here $\operatorname{Pr}$ cannot be defined in terms of $U$. In order to reflect the (explicit or implicit) quantification over trajectories in the semantic definition of the future operators above, a more suggestive notation is introduced in [BMP81, ClE81]. According to that notation, $G$ is $\forall G, X$ is $\exists X, F^{+}$is $\forall F, U$ is $\forall U, \operatorname{Pr}$ is $\forall P r, F$ is $\exists F, N$ is $\forall X$ and $G^{-}$is $\exists G$, and informally $\forall$ means in all possible trajectories and $G$, 
$X, F, U$ and $\operatorname{Pr}$ are interpreted as in the linear framework. However, therein none of the symbols $\forall, G, X, F, U$ and $\operatorname{Pr}$ denotes by itself an operator. By the way, note that the idea of breaking the operators $\forall G, \forall X, \forall F$, . in one branching operator $\forall$ and linear operators $G, X, F, \ldots$ (that appears in [EmH82, EmH86]) may be identified as one of the reasons for the introduction of the Ockhamist approach in the programming area.

Some examples of branching Peirceans formalisms (pbranch for short) follow: the tense system $\mathrm{K}_{6}$ is $\operatorname{pbranch}(G, H)$; the tense system in [McC79] is pbranch $\left(G, H, F^{+}\right)$(note that therein it is not imposed the linearity of the past); the temporal logic in [SCS82] is pbranch $\left(G, H, F^{+}, Y\right)$; the branching logic referred in [Lam80] is pbranch $\left(G_{0}, F_{0}^{+}\right)$; the temporal logic UB in [BMP81] is pbranch $\left(G_{0}, N, F_{0}^{+}\right)$; and the temporal logic CTL, as was described in [CIE81], may be considered as $\operatorname{pbranch}\left(N, U_{0}, P r_{0}\right)$ (forgetting the labeling of $N$ by process numbers and taking into account that the primitive operator $\exists U_{0}$ therein considered may be defined in terms of the above operator $\operatorname{Pr}_{0}$ ).

The Ockhamist approach introduces a semantics based in situations of the form $(\mathscr{I}, \omega, t)$ where $\mathscr{I}$ and $\omega$ are as before and $t$ is a trajectory of $\mathscr{I}$ that passes through $w$ that is called the actual [Bur78] or prima facie [Pri67, Tho70, McA76] future of that particular situation (in [Tho70, McA76] philosophical reasons are given against the term "actual future" within this context). The addition of the actual trajectory is the main difference between the tense semantics according to the two approaches (although a difference appears in [Bur78] with respect to the mapping of truth values to the propositional symbols: therein those values are related with each possible future branch, and not only with each world). As a matter of fact, in both approaches we can define the notions of truth, validity and semantic entailment as described in Section 1.1. The Ockhamist semantics is less intuitive from our point of view, since we basically agree with [McC76] in that in a nondeterministic universe there is no reason for choosing a particular (although arbitrary) trajectory for the actual future.

The Ockhamists formalisms usually contain one, or more branching operators and may include any set of linear operators. The satisfaction of a linear operator by a situation $(\mathscr{I}, \omega, t)$ is defined in a similar way to that used in the linear formalisms within the trajectory $t$. For instance, if $G$ denotes the "always in the future" linear operator, then

$$
(\Phi, w, t) \equiv(G f) \text { iff }\left(\forall w^{\prime}\right)\left(\left(w \mathscr{R} w^{\prime} \wedge w^{\prime} \in t\right) \Rightarrow\left(\mathscr{I}, w^{\prime}, t\right) \models f\right)
$$

A few branching operators have been considered in these formalisms. Note that without the branching operators the Ockhamists formalisms collapse into linear formalisms. For instance, the system OT defined in [McA76] includes the "always in the future" branching operator, denoted below by $A$ ( $L$ in [McA76]), defined as follows:

$$
(\mathscr{H}, w, t)=(A \not) \text { iff }\left(\forall t^{\prime}\right)\left(\forall w^{\prime}\right)\left(\left(w \mathscr{R} w^{\prime} \wedge w^{\prime} \in t^{\prime}\right) \Rightarrow\left(\mathscr{I}, w^{\prime}, t^{\prime}\right) \vDash \not\right)
$$

The system OT belongs to the formalism $\operatorname{obranch}(A ; G, H)$ (Ockhamist branching formalism using the branching operator $A$ and the linear operators $G$ and $H$ ).

Other much more interesting branching operator is the "in all trajectories" operator ("necessity" operator in [Pri67] and "inevitability" operator in [Tho 70]), $\forall$, already mentioned. Its semantics is defined as follows:

$$
(\mathscr{I}, \boldsymbol{\omega}, t) \vDash(\forall f) \text { iff }\left(\forall t^{\prime}\right)\left(\omega \in t^{\prime} \Rightarrow\left(\mathscr{I}, \omega, t^{\prime}\right) \vDash f\right)
$$

Returning to the concurrent programming area, we find formalisms of this type, 
with only the branching operator $\forall$, but considering other linear operators (besides $G$ and $H$ ) that have been shown to be useful in that application area. For instance, the logic proposed in [Nis80] is seen as $\operatorname{obranch}(\forall ; U)$ (being $\forall$ of the form $\llbracket \not k \rrbracket$ for $h$ a program). In this logic, that combines in some sense the dynamic logic with the usual temporal operators, the name state formulas is given to the formulas that contain no temporal operator or whose main operator is $\forall$.

In [EmH82, EmH86] the same classification of state formulas is adopted, being the other formulas called path formulas. The language CTL* defined in [EmH86] is seen as obranch $\left(\forall ; X, U_{0}\right)$ (being therein $A$ used instead of $\forall$ ) and the language in [EmH82] is seen as an alternative characterisation of the language CTL of [ClE81] that can be obtained as a sublanguage of CTL* by imposing some restrictions on the syntax of the formulas. Therein, the syntactic distinction between state and path formulas is also reflected at the semantical level: the satisfaction of state formulas is defined on situations of the form $(\mathscr{I}, \omega)$ and the satisfaction of path formulas on situations of the form $(\Phi, w, t)$. These situations are related in an obvious way.

Although this semantics seems more intuitive than the pure Ockhamist one (the price to be paid is a more complex syntax with the necessary distinction between state and path formulas), there is no real important difference between them (they lead to the same sets of true and valid formulas). By the way, a definition of $\mathrm{CTL}^{*}$ without distinguishing state and path formulas is found in [Sti87], although without recognising the close relationship to the Ockhamist approach. Therein, an axiomatisation for an $\operatorname{obranch}(\forall ; U)$ propositional language is proposed and proved to be complete (against a class of interpretation structures, of a form similar to that defined above, where all trajectories are infinite). For a complete axiomatisation for an $\operatorname{obranch}(\forall ; S, U)$ propositional logic see [Zan89] (although therein a slightly different semantics is considered).

The logic proposed in [LeS82] for reasoning about probabilistic programs may also be considered as obranch $\left(\forall ; X, U_{0}\right)$ if we interpret the "certainly" operator $\nabla$ proposed therein as meaning "for all paths" (see Section 14 in [LeS82]). The operator $\nabla$ was originally proposed in [LeS82] with the following informal meaning "for all paths in a set of paths of probability one", and its semantics is rigorously defined in the style of the Ockhamist approach, enriched with probability measures, where again no distinction is made between state and path formulas, being all formulas considered as path formulas.

It should also be noted that in order to drop the distinction between state and path formulas another approach is proposed in [HKP82]. Therein, a process logic (PL) is defined where all formulas are path formulas: the state formulas are seen as particular cases of path formulas whose truth value only depends upon the first state in the path. Like the logic in [Nis80], $P L$ also combines the dynamic logic operators with the usual temporal operators. Briefly, besides the usual propositional conectives, $P L$ introduces the unary operators $f$ and $[\not h]$ (where $h$ is a program) and the binary operator suf. Their semantics is defined on paths (finite or infinite sequences of states) as follows: $f f$ is satisfied by a path iff $f$ is satisfied in the first state of the path (seen as its prefix of length 1); suf corresponds to the until operator $U ;[h] f$ is satisfied by a finite path $\left\langle\Delta_{1}, \ldots, \Delta_{i}\right\rangle$ iff $f$ is satisfied by every finite path $\left\langle\sigma_{1}, \ldots, s_{r}\right\rangle$ (with $i \leq r$ ), or infinite paths $\left\langle\sigma_{1}, \ldots\right\rangle$, such that $\left\langle\sigma_{i}, \ldots, \sigma_{r}\right\rangle$ (respectively: $\left.\left\langle\sigma_{i}, \ldots\right\rangle\right)$ is a path assigned to the program $h([h] /$ is satisfied by every infinite path). Now, this semantics does not seem to be more intuitive than the previous one. Moreover, we do not like the need for having an operator like $f$. 
Finally, yet another approach is proposed in [Car85, CaS88a] that intends to separate completely, through appropriate operators, the assertions about each trajectory from the assertions about the tree structure. For that, this approach also considers an "in all trajectories" operator, herein designated by $L$ (and $M=\neg L \neg$ is its dual and means "in some trajectory"), but defines its semantics through situations of the form $(\mathscr{I}, \omega)$ in a good Peircean style (and so all the formulas may be considered as state formulas). The semantics of the other temporal operators is the usual one in such Peircean formalisms. The semantics of $L$ takes into account that a linear structure is a particular tree structure with only one trajectory, and it is rigorously defined as follows (where $\mathscr{I}=$ $(\mathscr{W}, \mathscr{R}, \mathscr{T}, \mathscr{M}))$ :

$(\mathscr{I}, \omega) \vDash(L f)$ iff $(\forall t)(\omega \in t \Rightarrow(\mathscr{H}[t], \omega) \vDash f)$

where $\mathscr{I}[t]=(t, \mathscr{R} \uparrow t,\{t\}, \mathcal{M} \uparrow t)$, that is, intuitively, $\mathscr{I}[t]$ is the interpretation structure corresponding to the tree with only one trajectory: $t$. Informally, $(L f)$ means that $f$ is true in the present, whatever the future will be. But, contrarily to what happens within the Ockhamist approach, here "whatever the future will be" means that we consider separately the different future alternatives once forever.

In the presence of the operator $L$, the meaning of the other temporal operators of the language depends upon if they are within its scope, or not. Outside the scope of $L$ the other temporal operators behave like branching operators. Inside the scope of an $L$ they behave like linear operators. Hence, as a matter of fact, we can visualise $L$ as a meta-linear operator, since we may read $(L f)$ as "in the underlying linear framework $f$ holds". On the other hand, we may see $L$ as an operator that allows us to combine a pbranch (...) logic with the correspondent linear (...) logic in the scope of a pbranch $(L, \ldots)$ logic, in such a way that if $f$ is a formula without any $L$ then: 1) $f$ is valid in the pure branching logic pbranch (...) iff $f$ is valid in the combination logic pbranch $(L, \ldots)$; and 2$) f$ is valid in the linear logic iff $(L f)$ is valid in the combination logic (these and other relationships are discussed in [CaS89a]). Note that this combination logic is particularly elegant when the semantic definition of the branching operators (besides $L$ ) does not need quantifications over the trajectories (as is the case of all the past operators and of the future operators $G$ and $X$ ), since in that case their semantic definition coincides in both the linear and the pure branching logic that we want to combine. As we shall see in Section 4, the family of logics we consider for supporting the layered methodology at hand satisfies this requirement.

The basic idea under the semantic definition of $L$ is to change the frame of the semantic analysis from the branching structure to the underlying linear structure. This frame change character of the operator $L$ is a novelty of this operator that has some consequences, both on its semantics and on its axiomatisations and their completeness proofs. For instance, with respect to the semantics, contrarily to what it is usual, this operator does not even preserve the truth in a frame (although it obviously preserves the validity). To see this it is enough to consider the frame $\mathscr{F}=(\mathscr{W}, \mathscr{R}, \mathscr{T})$ corresponding to the tree in Fig. 1 (where it is assumed that $\mathscr{R}$ is the transitive closure of the relation defined by the arrows and that the trajectories are the maximal $\mathscr{R}$-chains on $\mathscr{W}):$ we have $\mathscr{F} \models H(p \wedge$ $\neg p) \Rightarrow F G(p \wedge \neg p)$, but $L(H(p \wedge \neg p) \Rightarrow F G(p \wedge \neg p))$ it is not true in $\mathscr{F}$, since this formula is not satisfied in the initial world $w 1$.

Although frame change operators are not usually considered, we believe that different operators of this kind may be useful in many applications. As an 


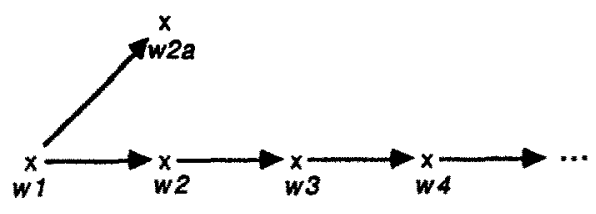

Fig. 1

illustration, assume that we are using a linear logic to describe the behaviour of a society of animated objects [SFS88, Fia89], where the time structure is identified with a possible sequence of events occurring in the lives of the different objects. Then we may wish to consider an unary frame change operator []$^{o}$ for each object $o$, with the following informal semantics (where we write $[f]^{\circ}$ instead of $\left([]^{\circ} f\right)$ ): $[f]^{\circ}$ asserts that $f$ holds in the current state of object $a$, according to its life (that is, according only to the sequence of the events in its life). Then identifying, to simplify, the interpretation structure $\mathscr{F}$ with a possible sequence of events for the whole society life, and identifying the current world/state with the sequence of events that have already occurred, we can define the semantics of such an operator as follows:

$$
(\mathscr{\Phi}, w) \vDash[f]^{o} \text { iff }\left(\mathscr{I}_{0}, w_{0}\right) \vDash \mathscr{f}
$$

where $\mathscr{I}_{o}$ and $\omega_{o}$ are the projections of the sequences of events $\mathscr{I}$ and $w$ on the set of all possible events that may occur in the life of the object $o$ (that is, informally, we obtain the sequences $I_{o}$ and $\omega_{o}$ by taking out from the sequences $\mathscr{I}$ and $w$ all the events that are not associated with the object $o$ ). Obviously, this possible frame change operator is herein only outlined and need further attention (namely concerning the difficulties that it probably introduces with respect to axiomatisations).

Returning to the operator $L$ and comparing it with the operator $\forall$, we may say that these operators look similar, although they behave differently. For instance, we have that $(\forall f \Leftrightarrow f)$ and $(L f \Leftrightarrow f)$ are both valid if $f$ contains no future operators. However, since $L$ really means "for all embedded linear time models", we have, unlike $\forall$, that any occurrence of $L$ within the scope of another $L$ is redundant. That is, more precisely, the following formula is valid: ( $L f \Leftrightarrow$ $L f^{-L}$ ), where informally $f^{-L}$ is $f$ taking out every $L$ (the formal definition is given later on), although some instances of $\left(\forall f \Leftrightarrow \forall f^{-\forall}\right)$ are not valid. For example, the formula $(\forall F \forall G p \Leftrightarrow \forall F G p$ ) is not satisfied in the initial world $w 1$ of the tree in Fig. 2 (where the trajectories are the maximal $\mathscr{R}$-chains on $\mathscr{W}$ ), since when we analyse the satisfaction of $\forall F \forall G p$ in some world, the future worlds where $\forall G p$ must be satisfied, at least one in each trajectory, still may have different possible future trajectories (contrarily to what happens when we analyse the satisfaction of $L F L G p$ ).

The operator $\forall$ is more powerful (from the expressibility point of view), since it allows us to pass from the branching level to the linear level (of the trajectories) and to return to the branching level. This is possible since its semantics is based on situations that combine in only one structure both the linear and the branching levels. On the other hand, the operator $L$ has a more intuitive semantics (from our point of view), although it only allows us to pass from the branching level to the linear level. However, as we shall see in Sections 3 and 4 , in some cases the opposite move from the latter to the former is not necessary and this apparent handicap of the operator $L$ becomes an advantage, since it allows the identifiction 


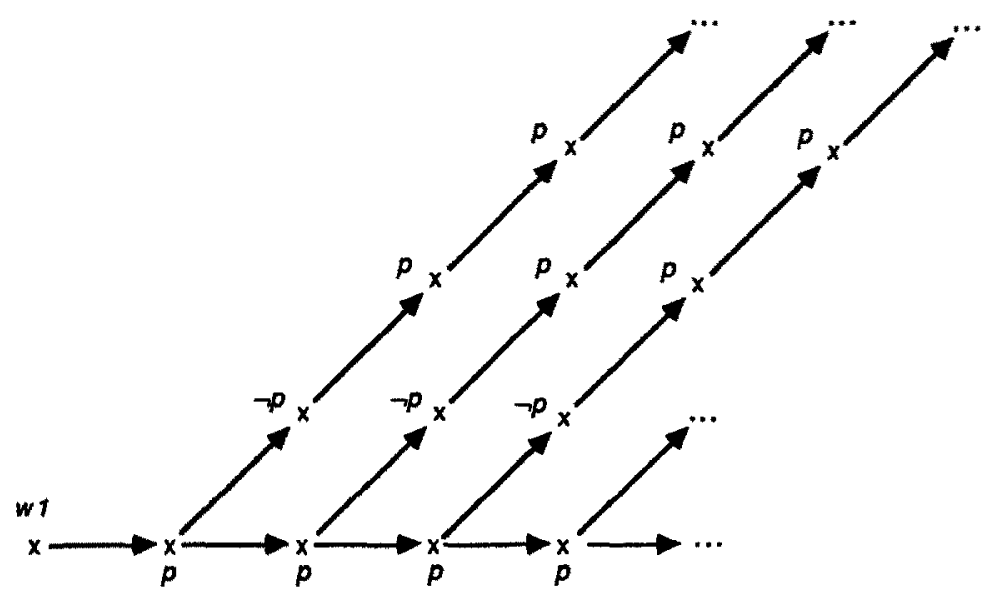

Fig. 2

of the formulas that express linear properties by examining only their first operator (ignoring their structures). As a matter of fact, any formula of the form $L_{f}$ expresses a linear property, but that is not always the case with respect to the formulas of the form $\forall \neq$.

It should also be stressed that, as it was already pointed out in [Pri67], p. 130, we can see the Peirceans formalisms as fragments of Ockhamists formalisms (including the $\forall$ operator) defined by imposing appropriate syntactic restrictions on the formulas of the latter. This strategy is adopted in [EmH86] in order to compare the different expressiveness powers of different linear and branching logics usually considered in the concurrent programing area. Therein it is proved, in particular, that the conclusion of [Lam80] that the linear and the branching time have incomparable expressible powers cannot be accepted as a universal assertion, although it is true for the particular linear and branching logics considered in [Lam80] (that, as we have already mentioned, belong to the formalisms: linear $\left(G_{0}\right)$ and pbranch $\left(G_{0}, F_{0}^{+}\right)$). Moreover, in [EmH86] it is proved that the language CTL* is more expressible than both logics considered in [Lam80] (note that the same type of argument can show that a linear(...) logic is less expressible than the correspondent $p b r a n c h(L, \ldots)$ logic, although logics belonging to the formalisms, for instance, $\operatorname{pbranch}(L, G)$ and $\operatorname{pbranch}\left(F^{+}, G\right)$ have different expressive powers).

However, even if we accept the inclusion of the Peirceans formalisms in the Ockhamists formalisms in order to provide a uniform formalism where we can compare the expressive powers of different logics, we do not think that it is correct to defend the use of some fragment of an Ockhamist formalism instead of the correspondent Peircean formalism. As a matter of fact, not only the Peircean formalisms have a completely different underlying semantic approach, as well as we think that, whenever possible, we should avoid the definition of languages where the application of the operators is syntactically restricted. Otherwise, there would be no reason to argue against the use of an appropriate fragment of the language $C T L^{*}$, where all the formulas have the form $\forall f$, for $f$ without any $\forall$, instead of the corresponding linear logic (extreme position that no one has defended until now). 
Finally, we would like to remark that this overview does not exhaust the multitude of temporal logics that have been proposed in the literature for concurrent programming specification and verification. In particular, it should be stressed that recent efforts have been made for combining temporal operators with automata: for instance, [CGK89] defines (in a Peircean-like style, where all formulas are state formulas) one such mixed temporal logic that is claimed therein to be as expressive as CTL*. However, the analysis of such logics is beyond the scope of this paper.

\section{A Layered Methodology for Systems Specification and Verification}

In this section we briefly present the layered methodology for database and information systems specification and verification that was proposed in [CaS88a, CaS88b]. Examples and further details can be found therein. As mentioned in the Introduction, the approach includes five layers, all of them independent of the target database model.

\subsection{The First Layer}

The first layer deals with the description of the static aspects of the system database: at this stage, we specify the information structure (that is, adopting for instance the Infolog terminology, we specify the relevant data types plus the entity types and their attributes), as well as the static constraints we want to impose on the stored information. Hence, in this layer we specify which are the allowed database states. A trivial example of a static constraint is "the balance of every bank account must be nonnegative".

\subsection{The Second Layer}

In the second layer we are able to impose constraints on the updating of the stored information without any commitment on how those updates will be performed. So, in this layer, we extend the first-layer specification with a set of transition constraints, that limit the possible sequences of database states without any reference to specific database-update operations (both static and transition constraints are usually called data integrity constraints). Simple examples of transition constraints are: "once a bank account exists, it exists forever", "salaries must not decrease during the same contract", "if someone ceases to work for a company, then that person can never work again for that company".

\subsection{The Third Layer}

Following [CCF82, FuN86], in the third layer (their second level, since they do not recognize the need for splitting the two classes of data integrity constraints into two different layers, as we do) we specify the update operations. These operations should be defined in such a way that no data integrity constraint is violated. The activities that the system will perform (to be specified in the fifth layer) should only use these update operations. 
According to the Infolog approach, both database-update and messageexchange operations are considered to be events. Thus, in this layer we introduce a finite set of (database) update event sorts, define their attributes and impose constraints on their values (event attribute constraints); moreover, we specify their effects on the stored information through change rules (that correspond to the pre and postconditions considered in the programming are [Hoa69]) and event scope constraints (delimiting the database attributes that may be modified by events of a given sort); finally, in order to guarantee that the update operations preserve the data integrity constraints, we impose enabling constraints on each update event sort (excluding the birth event sort), whenever necessary. For instance, in order to guarantee that an withdrawal account update preserves the static constraint given in Section 3.1, we impose that such an operation only can be performed when the balance of the account in the database is greater or equal than the amplitude of the withdrawal.

Also according to the adopted approach, we identify the system state with the sequence of all events that have already occurred (assumed to be all distinct), starting with the (system) birth event. Hence, the database state (the valuation of its attributes) is a function of the system state. Incidentally, this clear distinction between the system state and the database state allows specifications with high degrees of memory independence, as has already been mentioned in Section 2.

\subsection{The Fourth Layer}

In the fourth layer we specify the communication mechanisms of the system and impose constraints and requirements on its behaviour, without any commitment on how the system will fulfil them.

For this purpose we start by introducing a set of message-reception and message-emission event sorts. Their scope should be empty, since we assume that no message-exchange operation modifies the stored information (database state). Note that we also assume that a message is produced by the initiative of the source system and the destination system has no control whatsoever on the moment of its arrival. This allows a completely separation of the information transfer problems from the synchronisation problems [SCS82].

Moreover, we introduce at this layer behaviour safety constraints, as well as liveness requirements. Their importance for specifying reactive systems has already been well established in the concurrent programming area. Safety constraints state that "something bad never happens". Liveness requirements state that "something good must happen" and have the specific form: "if some condition $f$ becomes true, then sometime in the future $f^{\prime}$ will become true" (see e.g. [Pnu77, MaP81, OwL82]). For instance, considering a banking application again, a typical liveness requirement related with the responsiveness of the system to the environment stimulus is: "whenever the system receives a request for an withdrawal, sometime later it will send an answer message (that can be positive or negative, depending on the balance of the account)", and a related behavior safety constraint is: "it will not be produced more than one answer for each withdrawal request". Note that the constraints of the previous layers are also safety constraints. Safety constraints of this fourth layer are called behaviour safety constraints.

A rather interesting but unusual type of behaviour safety constraints was identified in [CaS88a]. Such constraints are called FDP constraints since they 
state conditions where the future is (partially) determined by the present (where "present" means the current system state, that is, the sequence of all events that have already occurred until now, and not the current database state). As an example, consider the liveness requirement above. Different complying sequences of events may provide different kinds of answer to the same withdrawal request, depending on the rate the system will perform other update operations on the same account. However, we might wish to impose that: "the kind of answer (positive or negative) is determined by the prevalent conditions (the system state) at the moment of the reception of the withdrawal request". This FDP constraint is asserted without any commitment on how it will be fulfilled by the system activities, although it restricts the set of possible implementations. For instance, an implementation consisting of random processing the deposit and withdrawal requests does not enforce this FDP constraint, but a FIFO discipline corresponding to processing those requests by the order of their arrival does. It should be stressed that, even if we impose this FDP constraint, the system may still follow different trajectories, but all of them must provide the same kind of answer to an withdrawal request (obviously, different withdrawal requests may have different answers).

\subsection{The Fifth Layer}

In the fifth layer we specify the system behaviour (that is, the activities that the system should perform and their triggers) in such a way that all possible trajectories of the system life satisfy every constraint/requirement included in the previous layers. The fifth layer refines the fourth and we may see it as suggesting procedural implementation strategies.

According to the proposed approach, the behaviour of a system is specified by a behaviour rule. This rule usually includes only the triggering rules that specify the process sorts of the system and the synchronisation rules that impose some scheduling discipline on the concurrent execution of those processes. It should be stressed that a behaviour rule not only imposes that each possible trajectory of the system life must satisfy all the specified triggering rules (liveness requirement) and synchronisation rules (safety constraint), but also states the following safety causality behaviour constraint: "besides the system birth event and the message receptions (whose occurrence is not controlled by the system), an event can only occur in the system life if it has been generated according to one of the specified triggering rules".

The triggering rules have the general form:

$$
\text { (〈trigger }\rangle /\langle\text { guard }\rangle::\langle\text { transition-command }\rangle \text { ) }
$$

where the trigger is an event variable or the birth event constant, the guard is a condition whose truth value can be evaluated by the system at any state, and the transition command specifies the pattern of the reaction events. Informally, a triggering rule states that in reaction to the occurrence of a triggering event, producing a state where the guard is satisfied, sometime later the system shall generate a sequence of reaction events according to the pattern specified by the transition-command. This sequence of reaction events will in general be interleaved with events of other processes, besides a nonspecified number of message receptions. Note that a triggering rule does not impose any bound on how long it will take until all reaction events are generated. This corresponds to a form of 
unbounded nondeterminism that is only implementable assuming fairness hypotheses [GPS80, Lam80]. However, according to [SCS82], this form of nondeterminism is very common and desirable at the conceptual specification stage, since it allows the abstraction from the implementation issues related to the guarantee of fairness.

The transition commands are built from the atomic transition-commands using some of the standard forms of program composition (sequence, alternative and parallel composition), as well as other forms of composition useful for systems specification (a waiting composition and a kind of repetitive composition useful for processing batches). The formal definition of their syntax, as well as some examples, can be found in e.g. [Car85, CaS88b].

Each atomic transition command defines the generation mechanism of a reaction event and has the general form:

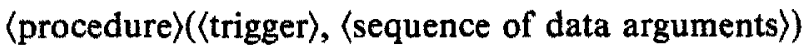

Each procedure maps a trigger event and a (possibly empty) sequence of data occurrences into a distinct reaction event whose cause is the trigger event. The events generated in this way are all distinct and the values of their attributes are defined by generation rules (usually equations) using only the trigger attributes and the data arguments of the procedure. In this way, the information in the database that is necessary for the generation of such events should be passed as data arguments of the procedure calls.

\section{Linear or Branching Formalisms?}

The choice between linear and branching formalisms has generated great controversy in the concurrent programming area (see e.g. [Lam80, EmH86]) and, in general, in any application this choice should precede the choice of the necessary temporal operators. Although we can always define a branching logic that is more expressive than a linear logic (using the "for all trajectories" operator defined in e.g. [EmH86], or the one herein proposed), that does not mean that a branching logic becomes a good choice for every application. The key idea is to choose for each application the simplest logic that does the job, that is, that is powerful enough to express all the constraints and requirements needed in that application.

In general, we may say that the linear formalisms are simpler than the branching formalisms and are appropriate whenever we only want to express properties that should be verified by every possible trajectory of the program (or system). On the other hand, we need to consider branching formalisms whenever we also want to state, for instance (see [Lam80, EmH86]): (1) the existence of some alternative (that is, the existence of a trajectory with some property); (2) the existence of different alternatives (that is, for instance, the existence of two trajectories satisfying different properties); or [CaS88a, CaS88b] (3) a constraint on the set of alternatives by a property that should be verified by that set of alternatives as a whole, and not only by each alternative separately (for instance, by imposing that some alternatives are incompatible).

Taking into account the previous ideas, [Lam80] stresses that linear formalisms are more appropriate for reasoning about concurrent programs and that branching formalisms are more appropriate for reasoning about nondeterministic algorithms that are implemented by concurrently executing all possibilities and stopping the entire computation if one succeeds (like nondeterministic automata). On the 
other hand, [EmH86] although agreeing that the linear formalisms are in general adequate for verifying the correctness of preexisting concurrent programs, stresses that in many situations there are also advantages in considering branching formalisms when dealing with concurrent programs (namely, for verifying that a concurrent program has a certain property by model checking and in automatic program synthesis from temporal logic specifications).

In the database area, the majority of the properties that we usually want to specify/verify are expressible using linear formalisms, as it is shown e.g. in [Kun84, LEG85, FiS86]. However, even in this area, properties exist that are only expressible using branching formalisms. That is the case of the FDP constraint presented in the previous section. Note that this constraint can be rephrased as follows: "whenever a bank receives an withdrawal request, then either all possible trajectories provide a positive answer to that request, or all possible trajectories provide a negative answer" (thus belonging to the third class of properties described above that are only expressible using branching formalisms). Taking into account the associated liveness requirement given in Section 3.4, we can also state this FDP constraint as follows: "whenever a bank receives an withdrawal request, if any of the possible trajectories provide a positive answer to that request, then all possible trajectories must provide a positive answer".

Finally, we end this section by illustrating how the mera-linear operator $L$ is useful for defining a unifying logic framework to support the layered methodology at hand. Only a sketch of such an unifying framework will be provided. The details can be found in [CaS88a] (where only semantic issues are considered) and in [CaS88b] (where both the semantic and the axiomatic approaches are developed).

Since it is not feasible to define a unique ideal temporal logic suitable for every layer (sufficiently abstract at the top layers and sufficiently detailed at the bottom layers), the unifying logic framework should be able to put together the best logic for each layer by linking them through convenient extension/refinement mechanisms. By the "best logic" for a layer we mean the simplest logic that is powerful enough to express all the constraints and requirements needed in that layer and that can be easily extended or refined in order to get the logic for the next layer. Note that since each layer specification must verify all the constraints and requirements included in the previous layer, from the expressibility point of view, the formal language chosen for each layer must be at least as powerful as the one chosen for the previous layer.

In what follows, each specification layer corresponds to a pair $(\mathscr{L}, A)$ where $\mathscr{L}$ is the chosen formal language and $A$ is the set of formulas (of $\mathscr{L}$ ) expressing the constraints and requirements of that layer. In the sequel $[(\mathscr{L}, A)]$ will denote the set of formulas (of $\mathscr{L}$ ) that are semantically entailed by $\boldsymbol{A}$ (for the axiomatic approach see [CaS88b]). Moreover, we say that a specification $\left(\mathscr{L}^{\prime}, A^{\prime}\right)$ complies with (or verifies) a specification $(\mathscr{L}, A)$ iff for each formula in $[(\mathscr{L}, A)]$ there is a formula in $\left[\left(\mathscr{L}^{\prime}, A^{\prime}\right)\right]$ with the same meaning (to be formally defined next).

As usual, in the first layer a specification is a pair $\left(\mathscr{L}_{1}, A_{1}\right)$ where $\mathscr{L}_{1}$ is a particular many-sorted first-order language with equality [End72] and $A_{1}$ is a set of first-order formulas expressing the static constraints.

Since the transition constraints express properties that should be verified by all possible sequences of database states, the discussion above shows that the linear formalisms are adequate for the second layer. On the other hand, with respect to the necessary temporal operators, according to what we have seen in Section 2.2 , it seems that the linear extension of $\mathscr{L}_{1}$ with the operators $U$ and $S$ 
is powerful enough to express the transition constraints (examples may be found in [CaS88a, CaS88b]). However, since in the next layers we need to talk about the events and since we identify each database state atomic transition with a distinct event occurrence, we adopt a richer language for the second layer that allows a better clarification of the forthcoming refinements. The language $\mathscr{L}_{2}$ proposed in [CaS88a, CaS88b] for the second layer is obtained by extending the language $\mathscr{L}_{1}$ of the first layer with: (1) the linear temporal operators $G$ and $H$; (2) the event sort symbol $e v$ (that abstractly denotes the set of all possible events, whatever they are, without attributes); (3) variables of this event sort; (4) the equality predicate symbol $=$ of sort $(e v, e v)$; and, finally, $(5)$ the unary eventoccurrence predicate symbol « 》 of sort $(e v)$.

The semantics of $\mathscr{L}_{2}$ is defined as usual (see Section 2.2). Note only that the domains of the different sorts, as well as the values associated to the variables, are state independent (implying the validity of the Barcan formulas [HuC68, McA76]), and that the interpretation of « 》 satisfies the following conditions: $M(\omega)(\ll \gg)$ is the singular subset of the event domain containing the event that has just occurred when looking to $w$ as the present system state; and $M(\omega)(\ll \gg)$ and $M\left(\omega^{\prime}\right)(\ll \gg)$ are disjoint for $\omega \neq w^{\prime}$, since we assume that no event occurs twice in a trajectory.

The formula $\ll \gg(t)$ (usually written as $\ll t \gg$ ) informally states that the event denoted by the term $t$ has just occurred. As it is discussed in detail in [FiS86], this possibility of reference to an event occurrence does not correspond to an explicit reference to the states (although it has some similarities). The event-occurrence predicate is present in all Infolog logics in order to describe the triggering mechanisms (see e.g. [SCS82, Car85, FiS86]) and it makes these logics very powerful, allowing the definition of operators like $X, Y, U$ and $S$ as nonprimitives. For instance, if $x$ is a variable (of sort $e v$ ) not occurring free in $f_{1} f_{1}$ and $f_{2}$, we can define:

$$
\begin{aligned}
& (Y f) \text { is }(\exists x)(P(\ll x \gg \wedge f) \wedge \neg P P \ll x \gg) \\
& (X f) \text { is }(\exists x)(F(\ll x \gg \wedge f) \wedge \neg F F \ll x \gg(\text { or }(\exists x)(\ll x \gg \wedge F(f \wedge Y \ll x \gg))) \\
& \left(f_{1} S f_{2}\right) \text { is }(\forall x)\left(\ll x \gg \Rightarrow P\left(f_{2} \wedge G\left(F \ll x \gg \Rightarrow f_{1}\right)\right)\right) \\
& \left(f_{1} U f_{2}\right) \text { is }(\forall x)\left(\ll x \gg \Rightarrow F\left(f_{2} \wedge H\left(P \ll x \gg \Rightarrow f_{1}\right)\right)\right)
\end{aligned}
$$

A second-layer specification is a pair $\left(\mathscr{L}_{2}, A_{2}\right)$, where $A_{2}$ is the union of $A_{1}$ with the set of formulas of $\mathscr{L}_{2}$ that expresses the transition constraints we want. It is obvious that $\left(\mathscr{L}_{2}, A_{2}\right)$ complies with $\left(\mathscr{L}_{1}, A_{1}\right)$, meaning that $\left[\left(\mathscr{L}_{2}, A_{2}\right)\right]$ contains $\left[\left(\mathscr{L}_{1}, A_{1}\right)\right]$.

The language $\mathscr{L}_{3}$ for the third layer is basically obtained from $\mathscr{L}_{2}$ by refining the event sort structure (syntactically and semantically), in order to introduce the database-update event sorts. A third-layer specification is a pair $\left(\mathscr{L}_{3}, A_{3}\right)$, where the set $A_{3}$ contains the event attribute constraints, the change and scope rules, and the enabling constraints. The former are described by first-order formulas and the others are specified using the operator $Y$ as described in e.g. [CaS88b]. $\left(\mathscr{L}_{3}, A_{3}\right)$ complies with $\left(\mathscr{L}_{2}, A_{2}\right)$ iff $\left[\left(\mathscr{L}_{3}, A_{3}\right)\right]$ contains $\left[\left(\mathscr{L}_{2}, A_{2}\right)\right]$, and in order to prove that result we only have to prove that $\left[\left(\mathscr{L}_{3}, A_{3}\right)\right]$ contains $A_{2}$.

In the fourth layer we must also refine the event sort structure in order to introduce the message-exchange event sorts. In addition, we are interested in two kinds of properties: properties that should be verified by all possible trajectories 
of the system life (that we shall call linear properties), such as the liveness requirements and the constraints enforced by the previous layer specification, and properties (called branching properties) that constrain the set of the possible trajectories for the system life by making some of them incompatible, such as the FDP constraints.

Hence, it is necessary to adopt in this layer some branching logic in order to express the branching properties. Moreover, it is desirable to also be able to pass from the branching level to the linear level (of the trajectories), whenever necessary, in order to express the linear properties and the complying relationship with the previous layer specification. Although this can be achieved with any of the "in all trajectories" operators $\forall$ and $L$, since we do not need to return from the linear level to the branching level, within this context, the operator $L$ has the advantages described in Section 2.3. Hence, in this layer we extend $\mathscr{L}_{3}$ with the operator $L$ (with the semantics described in Section 2.3).

A fourth-layer specification is a pair $\left(\mathscr{L}_{4}, A_{4}\right)$, where: $\mathscr{L}_{4}$ is basically obtained from $\mathscr{L}_{3}$ as it has been described (some predicates included in $\mathscr{L}_{4}$ according to [CaS88a, CaS88b], that respect to the causal relation between events, are not relevant herein); and $A_{4}$ is the union of $\left\{(L f): f \in A_{3}\right\}$ with the collection of formulas that express the requirements and constraints introduced in the fourth layer. Typically, the liveness requirements have the form $L\left(f_{1} \Rightarrow F f_{2}\right)$ (equivalent to $\left(f_{1} \Rightarrow L F f_{2}\right)$ whenever, as usual, no future operator occurs in $\left.f_{1}\right)$, the FDP constraints have the form $\left(f_{1} \Rightarrow\left(M f_{2} \Rightarrow L f_{2}\right)\right)$ (that is, $\left.\left(f_{1} \Rightarrow\left(L f_{2} \vee L \neg f_{2}\right)\right)\right)$, and the linear safety constraints appear as formulas where no future operator occurs or as formulas of the form $L\left(f_{1} \Rightarrow G f_{2}\right)$ (equivalent to $\left(f_{1} \Rightarrow L G f_{2}\right)$ if no future operator occurs in $f_{1}$, and equivalent to $\left(f_{1} \Rightarrow G f_{2}\right)$ if no future operator occurs in both $f_{1}$ and $f_{2}$ ).

A specification $\left(\mathscr{L}_{4}, A_{4}\right)$ complies with $\left(\mathscr{L}_{3}, A_{3}\right)$ iff $\left[\left(\mathscr{L}_{4}, A_{4}\right)\right]$ contains $\left\{(L f): f \in\left[\left(\mathscr{L}_{3}, A_{3}\right)\right]\right\}$. Note that although $(L f \Leftrightarrow f)$ is a valid formula for any $f$ in $A_{3}$, since the formulas in $A_{3}$ do not include future operators (and so we could also have considered $A_{3}$ instead of $\left\{(L f): f \in A_{3}\right\}$ in $\left.A_{4}\right)$, that may be not true for $f$ in $\left[\left(\mathscr{L}_{3}, A_{3}\right)\right]$. However, it is easy to prove that the inclusion of $\left\{(L f): f \in A_{3}\right\}$ in $A_{4}$ guarantees that $\left(\mathscr{L}_{4}, A_{4}\right)$ complies with $\left(\mathscr{L}_{3}, A_{3}\right)$.

When passing from the fourth to the fifth layer, we basically only need to extend the language $\mathscr{L}_{4}$ with a finite set of procedure symbols in the way described in e.g. [CaS88b] (note that the semantics proposed therein guarantees that no reaction event may occur before its trigger). In a fifth-layer specification $\left(\mathscr{L}_{5}, A_{5}\right)$, $A_{5}$ includes the change and scope rules included in $A_{4}$, plus the behaviour rule and the generation rules associated to the procedures. A specification $\left(\mathscr{L}_{5}, A_{5}\right)$ complies with $\left(\mathscr{L}_{4}, A_{4}\right)$ iff $\left[\left(\mathscr{L}_{5}, A_{5}\right)\right]$ contains $\left[\left(\mathscr{L}_{4}, A_{4}\right)\right]$, and in order to prove that we only have to prove that $\left[\left(\mathscr{L}_{5}, A_{5}\right)\right]$ contains $A_{4}$.

The fact that behaviour rules can be expressed in the language $\mathscr{L}_{5}$ is shown in e.g. [CaS88b] and will be omitted herein. However, as a related side comment, we would like to illustrate how an enrichment of this language with other frame change operator could simplify writing guards in the triggering rules involved in a behavior specification.

As a matter of fact, in order to fulfil the informal requirement that the guards must be conditions whose truth value can be evaluated by the system at any state (recall Section 3.5), restrictions are usually imposed on their syntax, namely that no future operator may occur in a guard (see e.g. [CaS88b]). However, some complex statements about the past could be more easily expressed if we could also use in the guards future operators, that should be analysed by the system, 
considering only the sequence of system-states from the initial system-state up to the current system-state (i.e. the sequence of events up to now). That is, we would have two logics: an internal logic of the evaluation of the guards by the system and an external logic, including the internal one, were we can express assertions about the system behaviour (similarly, in some sense, to what is done in [Gol82] with respect to the evaluation of the boolean expressions in specifying a sequential program behaviour, although therein no temporal aspects are obviously involved in the internal logic). This could be achieved by considering (in the external logic) a unary frame change operator [] with the following semantics (where we write $[f]$ instead of $([] f)$ ):

$$
(\mathscr{I}, w) \vDash[f] \text { iff }\left(\mathscr{I}_{w}, w\right) \vDash f
$$

where, according to the Peircean branching semantics we are considering, $\mathscr{I}=$ $(\mathscr{W}, \mathscr{R}, \mathscr{T}, \mathcal{M}), \mathscr{I}_{\omega}=\left(t_{\omega}, \mathscr{R} \uparrow t_{\omega},\left\{t_{\omega}\right\}, \mathcal{M} \uparrow t_{\omega}\right)$ and $t_{\omega}=\left(\left\{\omega^{\prime}: \omega^{\prime} \mathscr{R} \omega\right\} \cup\{\omega\}\right)$ (we could even omit the component $\left\{t_{\omega}\right\}$ in $\mathscr{F}_{\omega}$, since there is no interest in considering the operator $L$ in the guards).

Finally, it should be stressed that, as it is shown in [FiS86, FiS88], all the formulas in $A_{5}$ can also be expressed in an appropriate linear formalism. However, if we had chosen such formalism for this layer, then we could not rigorously prove that $\left(\mathscr{L}_{5}, A_{5}\right)$ complies with $\left(\mathscr{L}_{4}, A_{4}\right)$, since it would not be possible to prove that $\left(\mathscr{L}_{5}, A_{5}\right)$ verifies any FDP constraint included in the fourth layer (as it is illustrated in [CaS88b]).

\section{The Meta-Linear Operator L: Axiomatisation}

In this section we analyse the formal deductive aspects related with the operator $L$, within a propositional setting. The other primitive temporal operators we consider are $G$ and $H$ (and, as before, $M, F$ and $P$ denote the duals of $L, G$ and $H$ ). In the sequel, $\mathscr{L}$ denotes the language of the Peircean branching logic so obtained (and we use $\mathscr{L}_{1}$ whenever we want to refer to the language of the underlying linear logic, whose primitive temporal operators are only $G$ and $H$ ).

More precisely, we propose two axiomatisations for the language $\mathscr{L}$ : a finitary and an infinitary. Both axiomatisations are sound with respect to the semantics defined in Section 2.3 and the infinitary one is also complete with respect to that semantics (see Section 6). The possible completeness of the finitary axiomatisation is still an open question. The details and the proofs omitted herein can be found in [Car88].

\subsection{The Meta-Linear Operator L: Finitary Axiomatisation}

The set of axioms of the proposed finitary axiomatization for the language $\mathscr{L}$ is defined by the following axiom schemas:

(PC) every instance of a tautology is an axiom

(G1) $\quad G\left(f_{1} \Rightarrow f_{2}\right) \Rightarrow\left(G / f_{1} \Rightarrow G f_{2}\right)$

(H1) $\quad H\left(f_{1} \Rightarrow f_{2}\right) \Rightarrow\left(H f_{1} \Rightarrow H f_{2}\right)$

(GH1) $\quad \Rightarrow G P f$ 
(GH2) $\quad f \Rightarrow H F f$

(H2) $\quad\left(P f_{1} \wedge P f_{2}\right) \Rightarrow\left(P\left(f_{1} \wedge f_{2}\right) \vee P\left(f_{1} \wedge P f_{2}\right) \vee P\left(P f_{1} \wedge f_{2}\right)\right)$

(H3) $\quad P f \Rightarrow P(f \wedge H \neg f)$

(L1) $\quad L\left(\left(F f_{1} \wedge F f_{2}\right) \Rightarrow\left(F\left(f_{1} \wedge f_{2}\right) \vee F\left(f_{1} \wedge F f_{2}\right) \vee F\left(F f_{1} \wedge f_{2}\right)\right)\right)$

(L2) $\quad L(F(\neg / \wedge F G /) \Rightarrow F(\neg f \wedge G f))$

(L3) $\quad L\left(f_{1} \Rightarrow f_{2}\right) \Rightarrow\left(L f_{1} \Rightarrow L f_{2}\right)$

(L4) $\quad L f \Rightarrow M f$

(L5) $L f \Leftrightarrow L f^{-L}$

(L6) $\quad \Rightarrow L f$, if no future operator occurs in $f$ (that is, if $G$ does not occur in $f$ )

(L7) $\quad G L f \Rightarrow L G f$

(L8) $\quad H L f \Rightarrow L H f$

(L9) $\quad P L f \Rightarrow L P f$

(L10) $\quad M(G(p \wedge \neg p) \wedge f) \Rightarrow L(G(p \wedge \neg p) \Rightarrow f)$

(L11) LFFf $\Rightarrow F L F f$

and by the following rules of axiom generation:

(RG) if $f$ is an axiom, so is (Gf)

(RH) if $f$ is an axiom, so is $(H f)$

(RL) if $f$ is an axiom, so is $(L f)$

The only inference rule is "Modus Ponens" (MP): from $f_{1}$ and $\left(f_{1} \Rightarrow f_{2}\right)$ infer $f_{2}$.

The axioms and rules (PC), ., (H3), (RAG), (RAH) and (MP) are one of the different possible axiomatisations for the pure branching fragment of this logic (where no operator involves quantifications over the trajectories). This axiomatisation is based on the "Godel system" defined in [Boo79] and its completeness can be proved following the techniques in [Gab72].

With respect to the axioms related with the new operator $L$, some comments follow: (L1) states that in each trajectory the future is linear; (L2) guarantees that in each trajectory between any two future worlds it does not exist an infinite number of worlds; (L3) is the distributivity axiom of $L$ over $\Rightarrow$; (L4) states that for every world there is a trajectory passing through it; (L5) informally comes from the fact, already mentioned, that the operator $L$ allows us to move from the branching level to the linear level, but not to return to the branching level (note also that the formula $f^{-2}$ in this axiom can be inductively defined as follows: $p^{-L}$ is $p ;(\neg f)^{-L}$ is $\left(\neg f^{-L}\right) ;\left(f_{1} \Rightarrow f_{2}\right)^{-L}$ is $\left(f_{1}^{-L} \Rightarrow f_{2}^{L L}\right) ;(H f)^{-L}$ is $\left(H f^{-L}\right) ;(G f)^{-L}$ is $\left(G f^{-L}\right)$; and $(L f)^{-L}$ is $\left.f^{-L}\right)$; (L6) comes from the linearity of the past (there are no branches into the past); (L7), (L8), (L9) and (L11) are obvious and describe the relationships between $L$ and the other temporal operators (as we will see the converse of (L7) can be derived as a theorem); finally, (L10) states that there is at most one trajectory ending in each world. It should be stressed that we are not sure of the independence of (L10) and (L11) from the other axioms (within the context of the infinitary axiomatisation proposed next they do not need to be considered as axioms).

The reason why we adopted (RG), (RH) and (RL) as axiom generation rules instead of as inference rules is discussed in [CaS89a]. However, it should be stressed that it is trivial to prove that $(G f),(H \ell)$ and $(L \ell)$ are theorems whenever $f$ is a theorem, and so the axiomatisation is normal relatively to each of the three operators (see e.g. [Che80]). 
It is not difficult to verify that all the axiom schemes are valid (some semantic results useful for that can be seen in e.g. [CaS89a]). Hence, since the validity is preserved by the operators $L, G$ and $H$ and by "Modus Ponens" ( $G$ and $H$ even preserve the truth in an interpretation structure and "Modus Ponens" even preserves satisfaction), we have that the proposed axiomatisation is sound (and so also consistent).

If we extended the axiomatisation of the pure branching fragment of $\mathscr{L}$ $((\mathrm{PC}), \ldots,(\mathrm{H} 3),(\mathrm{RAG}),(\mathrm{RAH})$ and (MP)) with the following two axiom schemas:

$$
\begin{aligned}
& \left(F f_{1} \wedge F f_{2}\right) \Rightarrow\left(F\left(f_{1} \wedge f_{2}\right) \vee F\left(f_{1} \wedge F f_{2}\right) \vee F\left(F f_{1} \wedge f_{2}\right)\right) \\
& F(\neg f \wedge F G f) \Rightarrow F(\neg f \wedge G f)
\end{aligned}
$$

we obtain a sound and complete axiomatisation for the language $\mathscr{L}_{1}$ (whose semantics can be defined similarly to the semantics of $\mathscr{L}$, but omitting the component $\mathscr{T}$ from their frames and imposing that $\mathscr{R}$ is also right linear: see Section 2.2). Note that these axiom schemas are transformed into (L1) and (L2) when we axiomatise the mixed branching logic. Moreover, it is easy to see that a formula $L f$ is a theorem of the axiomatisation proposed for $\mathscr{L}$ iff $f^{-L}$ is a theorem of this axiomatisation for the underlying linear logic, and so the theorems of the form $L f$ can be obtained within the setting of this simpler linear axiomatisation (more general relationships between the axiomatisations proposed for $\mathscr{L}$ and $\mathscr{L}_{l}$ are established in e.g. [CaS89a]).

Besides the theorems of the axiomatisation for $\mathscr{L}$ that we can obtain using the previous result, many other theorems can be directly obtained. Some examples follow:

(a) if $\vdash f$, then $\vdash M f$

(b) $\quad-(L G f \Rightarrow G L f)$

(c) if no future operator occurs in $f$, then:

$$
\begin{aligned}
& \mapsto(L f \Leftrightarrow f) \quad \mapsto\left(L_{f} \Leftrightarrow M_{f}\right) \quad \mapsto\left(L\left(f \Rightarrow f^{\prime}\right) \Leftrightarrow\left(f \Rightarrow L f^{\prime}\right)\right. \\
& \mapsto\left(H L f \Leftrightarrow L H_{f}\right) \quad \mapsto(P L f \Leftrightarrow L P f) \quad r(G f \Leftrightarrow L G f) \\
& \mapsto(L F f \Rightarrow F L f) \\
& \text { (d) } \quad-L G(f \wedge \neg f) \Leftrightarrow G(f \wedge \neg f) \\
& \text { (e) } \quad-G(f \wedge \neg f) \Rightarrow(M f \Rightarrow L f)
\end{aligned}
$$

All of these results can be obtained without using the axiom schemas (L10) and (L11), with the exception of the last one that is proved using (L10). As an illustration, we show next how to derive (b).

(1) $L G f \Rightarrow G P L G f$

(2) $P L G f \Rightarrow L P G f$

(3) $P G F \Rightarrow f$

(4) $L P G f \Rightarrow L f$

(5) $P L G f \Rightarrow L f$

(6) $G P L G f \Rightarrow G L f$

(7) $L G f \Rightarrow G L f$
(GH1)

(L9)

(it is already a theorem of the tense axiomatisation $K_{f}$ )

(from 3 using e.g. the normality with respect to $L$ ) (tautologically from 2 and 4 )

(from 4 using e.g. the normality with respect to $G$ )

(tautologically from 1 and 6)

Taking into account this theorem and the axiom schema $(L 7)$, we have that $L$ can interchange with $G$ in both directions. On the other hand, in general $L$ can 
only interchange with $H$ and $P$ in one direction and cannot interchange with $F$, since some instances of forms $(L H f \Rightarrow H L f),(L P f \Rightarrow P L f),(F L f \Rightarrow L F f)$ and $(L F f \Rightarrow F L f)$ are not valid (examples can be easily found [Car88]).

Finally, note that if we intend to characterise only the interpretation structures where the set of the possibly terminal worlds coincides with the set of the terminal worlds, then we should extend this axiomatisation with the axiom schema $(M G(f \wedge \neg f) \Rightarrow G(f \wedge \neg f))$, since that class of interpretation structures coincides with the class of the interpretation structures where any of the formulas of this form is true. On the other hand, it is easy to see that, if no future operator occurs in $f$, then any formula of the form $((F H f \wedge G(H f \Rightarrow F H f)) \Rightarrow M G f)$ is true in any saturated interpretation structure, and that there are formulas of this form that are not valid.

\subsection{The Meta-Linear Operator L: Infinitary Axiomatisation}

Throughout the rest of this paper we assume the following abbreviations: false is $(p \wedge \neg p) ; 0$ is (Hfalse) (and identifies the initial world); $\left(P^{0} f\right)$ is $f$; and $\left(P^{n+1} f\right)$ is $\left(P\left(P^{n} f\right)\right)$, for any $n \geq 0$.

The set of axioms of the proposed infinitary axiomatisation for the language $\mathscr{L}$ is defined by the following axiom schemas:

(PC), (G1), (H1), (GH1), (GH2), (H2)

(H4) $\quad 0 \vee P 0$

(H5) $H f \Rightarrow H H f$

(L1) and (L3), ., (L9)

plus the axiom generation rules (RG), (RH) and (RL).

The inference rules are $(M P)$ and, for each $a \in A f m(\mathscr{L})$, the following infinitary inference rule:

(IR[a]) from $\left\{a\left[P^{n} 0\right]: n \geq 1\right\}$ infer $a[$ false $]$

where the set $\operatorname{Afm}(\mathscr{L})$ of the admissible forms for $\mathscr{L}$ is inductively defined as follows:

(i) the symbol "\#" belongs to $\operatorname{Afm}(\mathscr{L})$

(ii) if $a \in \operatorname{Afm}(\mathscr{L})$ and if $f$ is a formula $\mathscr{L}$, then $(G a),(H a),(L a)$ and $(f \Rightarrow a)$ also belong to $\operatorname{Afm}(\mathscr{L})$

and, when $a \in \operatorname{Afm}(\mathscr{L})$ and $f$ is a formula $\mathscr{L}, a[f]$ is the formula of $\mathscr{L}$ that we obtain by replacing the unique occurrence of \# in $a$ by $f$.

Briefly, we obtain this axiomatisation from the finitary one by removing the axiom schemas (H3), (L2), (L10) and (L11), and by introducing the axiom schemas (H4) and (H5) and the infinitary rules (IR $[a]$ ) (the axiomatisation so obtained is still normal with respect to all temporal operators). The derivation of (H3) and (L2) within this axiomatisation can be seen in [Car88], where some useful theorems and derived proof rules related with the infinitary inference rules (e.g. $\left.\left\{\left(f \Rightarrow P^{n} 0\right): n \geq 1\right\} \vdash \neg f\right)$ are also illustrated. The fact that (L10) and (L11) are also theorems is a consequence of the completeness of this axiomatisation. 
The proposed infinitary inference rules are based on a set of admissible forms whose definition is strongly influenced by the one given in [Gol82] within the context of an infinitary axiomatisation for the logic of sequential programming. However, since the infinitary axiomatisations are given herein in such a way that they verify the deduction metatheorem (strong version), the distinction between a logical system and its theories made in [Gol82] pp. 59 and 65-66, is irrelevant (even for completeness issues).

The soundness of this axiomatisation (with respect to the semantics defined in Section 2.3 ) is easily verified. Note that the infinitary rules even preserve the satisfaction by a situation (take into account that we have always only a finite number of past worlds).

On the other hand, if we extend the pure branching fragment of this axiomatisation ((PC), (G1), (H1), (GH1), (GH2), (H2), (H4), (H5), (RG), (RH), (MP) and (IR $[a]$ ), excluding ( $L a)$ of the set of admissible forms) with only the axiom schema (G2), we obtain an infinitary axiomatisation for the underlying linear logic (the infinitary linear axiomatisation for short). This axiomatisation is equivalent to the corresponding finitrary one, with respect to their theorems, although the former has a more powerful inference mechanism. Furthermore, the infinitary axiomatisations proposed for $\mathscr{L}$ and $\mathscr{L}_{i}$ are related similarly to the corresponding finitary axiomatisations.

\section{Completeness of the Infinitary Axiomatisation for $L$}

In this section we start by showing how the frame change character of the operator $L$ creates problems when we try to directly apply the usual Henkin construction of a verifying "model" (interpretation structure) to prove the completeness of any axiomatisation for $L$, and by describing one way to overcome those problems through an original and elegant extension of that construction (namely, by modifying the concept of world at hand). After we give a sketch of how this extended construction can be successfully applied to prove the completeness of the infinitary axiomatisation proposed for $L$. A more detailed outline can be found in [CaS89b].

In some steps of the construction we work in parallel with the logics and infinitary axiomatisations proposed for both the languages $\mathscr{L}$ and $\mathscr{L}_{1}$. For this reason, we use $\vDash_{l}$, instead of $\vDash$, within the scope of the semantics for $\mathscr{L}_{1}$, and we write $B_{l}, \ldots$ (resp. $f_{l}, \ldots$ ), instead of $B, \ldots$ (resp. $f, \ldots$ ), whenever we want to state explicitly that we are referring to a set of formulas (resp. a formula) of $\mathscr{L}_{l}$. Finally, we write " $B$ is consistent" and " $B$ is $m c$ " (resp. " $B_{l}$ is consistent" and " $B_{l}$ is $m c_{l}$ ") meaning that $B$ is, respectively, consistent and maximally consistent with the infinitary axiomatisation for $\mathscr{L}$ (resp. $\mathscr{L}_{i}$ ).

In order to prove the completeness of an axiomatisation we usually prove that if a formula is consistent with it then it is satisfiable (that is, there exists a situation where it is satisfied). The Henkin constructions based on the maximal consistent sets are very intuitive and achieve this sufficient condition by constructing an interpretation structure $\mathscr{I}$ for the language (e.g. the "canonical model" [HuC84]), whose worlds are maximal consistent sets (or, at least, are associated with maximal consistent sets) and that satisfies the following two conditions:

(i) the initial consistent formula that we want to prove that it is satisfiable (denoted in the sequel by $f_{i}$ ) belongs to some world in the frame;

(ii) $(\mathscr{I}, w) \vDash f$ iff $f \in w$, for any world $w$ in the frame and any formula $f$ of the 
language (or at least, using the filtration techniques, for $f$ belonging to some "well behaved" set of formulas, closed for subformulas and containing $f_{i}$, like the set of all subformulas of $\mathbf{f}_{\mathbf{i}}$ ).

Now, the previous construction cannot be directly applied to axiomatisations for languages involving the frame change operator $L$. Indeed:

(1) by definition, $(\mathscr{I}, w) \vDash(L \ell)$ iff $(\forall t \in \mathscr{T})(w \in t \Rightarrow(\mathscr{I}[t], w) \vDash f$;

(2) but, by (ii) above, $f \in \omega$ iff $(\Phi, \omega) \vDash f$;

(3) and, in general, if $f$ involves future operators we do not have $f \in \omega$ iff $(\mathscr{I}[t], w) \vDash f$, since we do not have $(\mathscr{I}[t], w) \vDash f$ iff $(\mathscr{I}, w) \vDash f)$.

Thus, we need to see how to characterise " $(\mathscr{I}[t], \omega) \vDash f$ " as a function of the formulas that belong to $w$. In order to be able to do that, we need to change the previous construction. The informal idea is the following:

(I) Define each world $w$ as a pair $\langle w .1, w .2\rangle$ such that:

$w .1$ is a $m c$ set that informally represents the formulas of $\mathscr{L}$ that are satisfied in $w$ according to the interpretation structure ("tree") $\mathscr{I}=(\mathscr{W}, \mathscr{R}, \mathscr{T}, \mathscr{M})$ to be defined (see Fig. 3);

and $w .2$ is a set of $m c_{l}$ sets, each representing the set of formulas of $\mathscr{L}_{l}$ that are satisfied in $\omega$ according to some trajectory $t$ that passes through $w$ (that is, according to $\mathscr{F}[t])$.

According to this idea: $\mathscr{M}$ should be defined as follows " $\mathcal{M}(\omega)(p)=1$ iff $p \in \omega .1$ "; the relation $\mathscr{R}$ between the worlds in the tree should only depend on the formulas that belong to their first component; and we must have

(*) ( $\left.L f_{l}\right)$ belongs to $w .1$ iff $f_{1}$ belongs to all sets in $w .2$

Moreover, in order to achieve (ii) above, $\Re$ should be defined in such a way that we can prove (using the usual techniques) that

(**) for every formula $f_{1}$ of $\mathscr{L}$ :

if $(\mathscr{I}, \omega) \vDash L f$ iff $L f \in \omega .1$, for all worlds $w \in W$ and (at least) all subformulas $L f$ of $f_{1}$, then $(\mathscr{I}, w) \vDash f_{1}$ iff $f_{1} \in w .1$, for all worlds $w \in \mathscr{W}$

and, obviously, $\mathscr{R}$ should satisfy the constraints imposed on the interpretation structures for $\mathscr{L}$

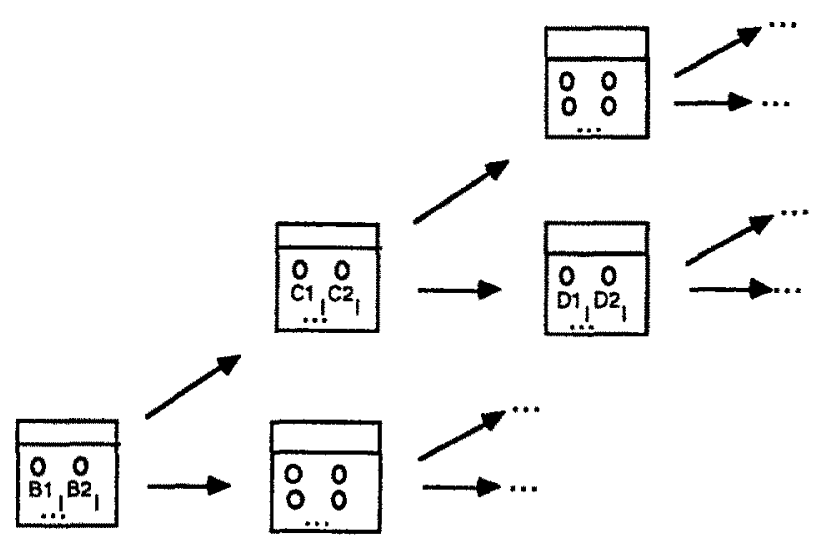

Fig. 3 
If $\mathrm{f}_{\mathbf{i}} \in \boldsymbol{w} .1$ for some $w \in \mathcal{W}$, then the completeness proof will be completed if we can prove the hypothesis of $\left(^{* *}\right)$ at least for $f_{1}=\mathbf{f}_{\mathrm{i}}$. This is achieved by the next two steps, taking into account $\left(^{*}\right)$ and the theorem $L f \Leftrightarrow L f^{-L}$.

(II) Define the set of trajectories $\mathscr{T}$ in such a way that:

(a) we can associate to each trajectory $t$ (at least) one interpretation structure of $\mathscr{L}_{l}, \mathscr{I}_{l}=\left(\mathscr{W}_{l}, \mathscr{R}_{l}, \mathscr{M}_{l}\right)$, satisfying:

$W_{l}$ is formed by one (and only one) element $B_{l}$ of $\omega .2$ (denoted below by $\operatorname{lin}(\omega)$ ), for each $w \in t$ (see Fig. 4)

$(\mathscr{I}[t], w) \models f_{l}$ iff $\left(\mathscr{I}_{l}, \operatorname{lin}(w)\right) \models{ }_{l} f_{l}$, for every $w \in t$ and every formula $f_{l}$

(b) and, for each $w$ in the tree (that is, for each $\omega \in W$ ) and each $B_{1} \in w .2$, there exists $t \in \mathscr{T}$ such that $B_{l}$ is a world of $\mathscr{I}_{l}$ (that is, $B_{l} \in \mathscr{W}_{l}$ ), defined as in (a).

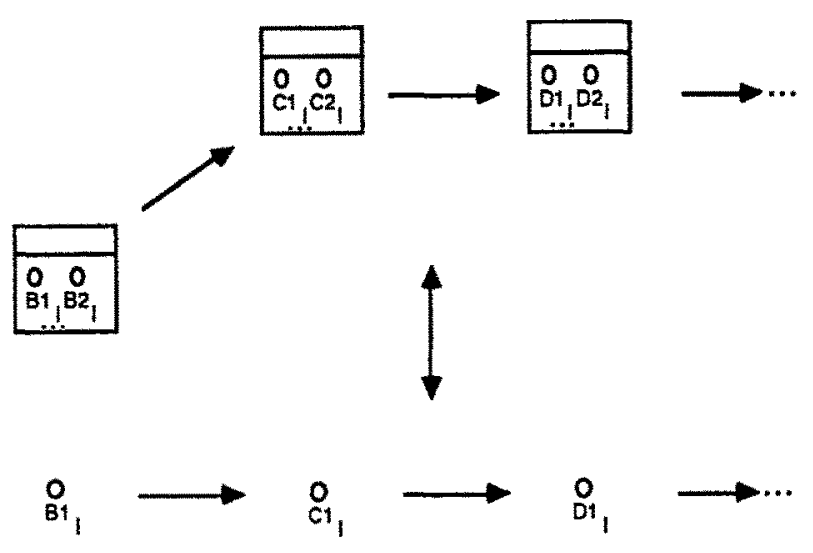

Fig. 4

(III) Prove, for the interpretation structures $\mathscr{I}_{l}=\left(\mathscr{W}_{l}, \mathscr{R}_{l}, \mathscr{M}_{l}\right)$ of $\mathscr{L}_{l}$ obtained according to (II), that

" $\left(I_{l}, B_{l}\right)=F_{i} f_{l}$ iff $f_{l} \in B_{l}$ ", for every $B_{l} \in W_{l}$ and every $f_{l}$ belonging to some relevant "well behaved" set of formulas of $\mathscr{L}_{I}$

using the usual Henkin techniques and the underlying linear axiomatisation.

In the sequel, the previous ideas are stated precisely and a sketch of the completeness proof of the proposed infinitary axiomatisation for $L$ is given. Note that part of the construction at hand could also be developed within the context of the proposed finitary axiomatisations: that is the case of all the results that are related with (I) above. However, we have not yet succeeded in achieving (II) and (III) above within the setting of those finitary axiomatisations.

We begin by constructing an initial "model" $\mathscr{I}$ for the language $\mathscr{L}$, that is later on refined, where we do not take care of its component $\mathscr{T}$ : the purpose is only to define the set of worlds and the accessibility relation in such a way that they fulfil the conditions described in (I). 


\section{Definition}

(a) $\mathscr{W}^{\circ}=\left\{\left\langle B, \operatorname{total}_{l}(B)\right\rangle: B\right.$ is $\left.m c\right\}$, where $\operatorname{total}_{d}(B)$ denotes the set formed by all $m c_{l}$ sets $B_{l}$ that satisfy the following condition:

$\left\{M\left(\wedge C_{l}\right): C_{l}\right.$ is a finite subset of $\left.B_{l}\right\} \subset B$

(Note that this condition is equivalent to $\left\{f_{l}:\left(L f_{l}\right) \in B\right\} \subset B_{l}$, whenever $B$ is $m c$ and $B_{l}$ is $m c_{l}$. Note also that, since $C_{l}$ is finite, $\left(\wedge C_{l}\right)$ is defined as usual in the scope of the Propositional Calculus. Hence, $\left(\wedge\left\{f_{t}\right\}\right)=f_{1}$ and $(\wedge \varnothing)=$ $\neg$ false).

(b) $\mathscr{R}^{0}$ is the binary relation over $\mathscr{W}^{0}$ defined as follows (where $w$ and $w^{\prime}$ belong to $\left.W^{0}\right): w \mathscr{R}^{0} w^{\prime}$ iff for all formulas $f$, if $(G f) \in w .1$ then $f \in w^{\prime} .1$

(c) $\mathcal{M}^{0}(\omega)(p)=1$ iff $p \in \omega .1$

Before proceeding, some useful notation follows.

\section{Notation}

(a) If $B$ is consistent, then $B^{\infty}$ is a particular $m c$ extension of $B$ (definable similarly to [Gol82, pp. 73-74]). Analogously, we define a $m c_{l}$ extension $B_{l}^{\alpha t}$ for $B_{l}$ consistent $t_{l}$.

(b) $\operatorname{PS}(\omega, f)=\left\langle B\right.$, total $\left.l_{l}(B)\right\rangle$, where $B$ is $\left(\left\{f^{\prime}:\left(H f^{\prime}\right) \in \omega .1\right\} \cup\{f\}\right)^{\infty}$, and $\operatorname{PS}(\omega, f)$ is the "past subordinated to $w$ according to $f$ ". The future subordinated FS $(\omega, f)$ is defined analogously.

(c) $\operatorname{LS}\left(\omega, f_{l}\right)=\left(\left\{f_{1}^{\prime}:\left(L f_{1}^{\prime}\right) \in \omega .1\right\} \cup\{f,\}^{\infty}\right.$, and we may read $\operatorname{LS}\left(\kappa^{\prime}, f\right)$ as the "linear world subordinated to $w$ according to $f$ ".

(d) If $B$ is $m c$ (resp. if $B_{l}$ is $m c_{l}$ ) then $\mathbf{n}(B)$ (resp. $\mathbf{n}\left(B_{l}\right)$ ) denotes the unique nonnegative integer $n$ such that $\left(P^{n} 0 \wedge \neg P^{n+1} 0\right)$ belongs to $B$ (resp. $\left.B_{l}\right)$.

It should be stressed that the existence of such $n$ in (d) above is one of the results in this completeness proof that we cannot prove within the scope of the finitary axiomatisations. As we shall see, such an existence will be particularly useful later on to allow us to describe the possible next worlds of each world w, and not only its possible future worlds. Note that $\mathbf{n}(B)$ represents, informally, the number of past worlds that a world built up from $B$ can have.

The initial "model" $\left(\mathscr{W}^{0}, \mathscr{R}^{0}, \mathscr{M}^{0}\right)$ is almost what we want. As a matter of fact, we can easily prove that $\left(\mathscr{W}^{0}, \mathscr{R}^{0}, \mathcal{M}^{0}\right)$ verifies conditions $\left({ }^{*}\right)$ and $\left(^{* *}\right)$ of (I), taking into account the next result.

\section{Proposition}

(a) If $\left(M f_{t}\right) \in w .1$ then $L S\left(w, f_{1}\right) \in w .2$.

(b) If $w \mathscr{R}^{0} w^{\prime}$ and if $(H f) \in w^{\prime} .1$ then $f \in w .1$

(c) If $\neg(H f) \in w .1$ then $\omega^{\prime}=\operatorname{PS}(w, \neg f) \in W^{\circ}, w^{\prime} \mathscr{R}^{0} w$ and $\neg f \in w^{\prime} .1$ (resp. if $\neg(G f) \in w .1$ then $w^{\prime}=\mathbf{F S}(w, \neg f) \in W^{0}, w^{0} w^{\prime}$ and $\left.\neg f \in w^{\prime} .1\right)$

Furthermore, we can prove that in order to guarantee that the accessibility relation satisfies all the constraints imposed on the intrepretation structures for $\mathscr{L}$ it is only necessary to take out of the model possible existent disconnected trees. So, we refine the initial model by restricting the set of worlds to those that are related 
with some world that contains the initial formula $\mathbf{f}_{\mathbf{i}}$ in its first component, or with worlds related with it.

\section{Definition}

(a) $W=\left\{w: w \in W^{0}\right.$ and $\left(w=w^{0}\right.$ or $\left.\left.w^{0} \mathscr{R}^{0} w\right)\right\}$, where $\mathbf{w}^{0}=\left\langle\left\{\mathbf{f}_{\mathbf{i}}\right\}^{\infty}, \operatorname{total}_{l}\left(\left\{\mathbf{f}_{\mathrm{i}}\right\}^{\infty}\right)\right\rangle$, if $0 \in\left\{\mathbf{f}_{\mathbf{i}}\right\}^{\infty}$, and $\mathbf{w}^{0}=\operatorname{PS}\left(\left\langle\left\{\mathbf{f}_{\mathrm{i}}\right\}^{\infty}, \operatorname{total}_{l}\left(\left\{\mathbf{f}_{i}\right\}^{\infty}\right)\right\rangle, \mathbf{0}\right)$, if not

(b) $\mathscr{R}=\mathscr{R}^{0} \uparrow W$

(c) $\mathscr{M}=\mathscr{M}^{0} \uparrow W$

According to what we saw at the beginning of this section, in order to finish the completeness proof at hand we only need to define a set of trajectories $\mathscr{T}$ in such a way that we can fulfil the conditions described in (II) and (III). For the purpose we need to refine the natural candidate $\mathscr{R}_{1}^{0}$ for the linear accessibility relation over the set $\mathscr{W}_{l}$ of the $m c_{l}$ sets:

$$
B_{l} \mathscr{R}_{l}^{0} B_{l}^{\prime} \text { iff for all formula } f_{l} \text {, if }\left(G f_{l}\right) \in B_{l} \text { then } f_{l} \in B_{l}^{\prime}
$$

For that refinement we use, in particular, the usual filtration techniques and we need some more notation.

\section{Notation}

(a) $w^{\prime}\left(\omega^{\prime}, \ldots\right)$ denotes a generic element of $W$ and $w_{1}\left(\omega_{1}^{\prime}, \ldots\right)$ denotes a generic element of $W_{l}$ (that is a $m c_{l}$ set).

(b) $\operatorname{subf}(f)$ denotes the set of all subformulas of $f$.

(c) $L$ subf $(f)=\left\{f_{l}\right.$ : exists $f^{\prime}$ such that $\left(L f^{\prime}\right) \in \operatorname{sub}(f)$ and $\left.f_{1} \in \operatorname{subf}\left(f^{\prime-L}\right)\right\}$.

(d) $\neg L \operatorname{subf}(f)=\left(L \operatorname{subf}(f) \cup\left\{\left(\neg f_{1}\right): f_{1} \in L \operatorname{subf}(f)\right\}\right)$.

Noting that $\operatorname{Lsubf}\left(\mathrm{f}_{\mathrm{i}}\right)$ is the set formed by the linear formulas that are relevant, we define the relation $\mathscr{R}_{l}$ in which we are interested as follows.

\section{Definition}

(a) $w_{i} \mathscr{R}_{i}^{\prime} w_{i}^{\prime}$ iff for every formula $f_{i}$ :

if $\left(G f_{1}\right) \in L \operatorname{subf}\left(\mathbf{f}_{i}\right)$ and $\left(G f_{1}\right) \in w_{1}$ then both $\left(G f_{i}\right)$ and $f_{l}$ belong to $w_{i}^{\prime}$ and if $\left(H f_{i}\right) \in L s u b f\left(f_{i}\right)$ and $\left(H f_{l}\right) \in w_{i}^{\prime}$ then both $\left(H f_{l}\right)$ and $f_{l}$ belong to $w_{l}$

(b) $\omega_{l} \mathscr{R}_{1} \omega^{\prime}$ iff $\left(\omega_{1} \mathscr{R}_{l}^{\prime} \omega_{i}^{\prime}\right.$ and $\left.\mathbf{n}\left(\omega_{l}\right)<\mathbf{n}\left(\omega_{l}^{\prime}\right)\right)$

Finally, introducing again some more notation, we define the desired set of the trajectories $\mathscr{T}$ and we finish the completeness proof (appealing to some omitted preliminary results).

\section{Notation}

(a) $\mathbf{n}\left(F, f_{1}, w_{1}\right)$ it is defined iff $\left\{i: i>0\right.$ and $\left.F\left(f_{1} \wedge\left(P^{i} 0 \wedge \neg P^{i+1} 0\right)\right) \in w_{l}\right\}$ is nonempty, in which case $\mathrm{n}\left(F, f_{1}, w_{l}\right)$ is the minimum of that set. 
(b) $G 1\left(w_{1}\right)=\left\{f_{i}: f_{1} \in \neg L s u b f\left(f_{i}\right)\right.$ and $G\left(\left(P^{i+1} 0 \wedge \neg P^{i+2} 0\right) \Rightarrow f_{1}\right) \in w_{1}$ for $i=$ $\left.\mathbf{n}\left(\omega_{i}\right)\right)$.

(c) $G 2\left(\omega_{l}\right)=\left\{F\left(\neg f_{1} \wedge\left(P^{i} 0 \wedge \neg P^{i+1} 0\right)\right)\right.$ : $G f_{h} \in L s u b f\left(f_{i}\right)$ and $\neg G_{f_{l} \in \omega_{l}}$ and $i=$ $\left.\mathbf{n}\left(F, \neg f, w_{l}\right)>\mathbf{n}\left(\omega_{1}\right)+1\right\}$

(d) If $(G$ false $) \in \omega_{1}$ then $\operatorname{next}\left(\omega_{1}\right)=P^{i+1} 0 \wedge \neg P^{i+2} 0 \wedge\left(\wedge G 1\left(\omega_{1}\right)\right) \wedge\left(\wedge G 2\left(\omega_{1}\right)\right)$, for $i=\mathbf{n}\left(\omega_{i}\right)$. Otherwise next $\left(\omega_{l}\right)$ is not defined.

Informally: $G 1\left(\omega_{1}\right)$ indicates which are the relevant linear formulas (and their negations) that must be part of the next (linear) world of $\omega$, according to any linear trajectory where $\omega_{1}$ may be embedded; and next $\left(\omega_{1}\right)$ characterises the formula that must be satisfied in the next (linear) world of $w_{1}$ according to the linear trajectories where $\omega_{1}$, will be embedded. Note that the inclusion of $\wedge G 2\left(\omega_{1}\right)$ in next $\left(\omega_{l}\right)$ will guarantee that if some relevant linear formula (or its negation) must be satisfied in some future world of $\omega_{l}$, that is not its next world, than that formula will be satisfied in some next world of ... of the next world of $w_{1}$. Note also that if $G$ false $\notin \omega_{1}$ then $F \operatorname{next}\left(\omega_{1}\right) \in \omega_{1}$.

\section{Definition}

Suppose that $w \in W$ and $w_{1} \in w .2$. Then:

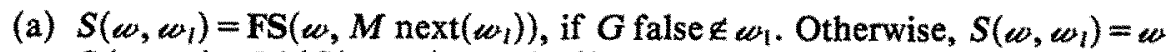

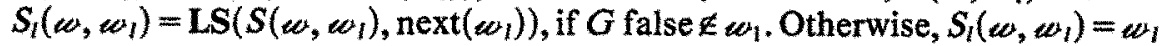

(b) $S^{0}\left(\omega, \omega_{1}\right)=\omega$ and $S_{1}^{0}\left(\omega, w_{1}\right)=\omega_{1}$ $S^{n+1}\left(\omega, \omega_{i}\right)=S\left(S^{n}\left(\omega, \omega_{i}\right), S_{l}^{n}\left(\omega, \omega_{i}\right)\right)(n \geq 0)$

$S_{i}^{n+1}\left(\omega_{,}, \omega_{l}\right)=S_{l}\left(S^{n}\left(\infty, \omega_{l}\right), S_{i}^{n}\left(\infty_{,}, \infty_{l}\right)\right)(n \geq 0)$

(c) $\mathbf{t}\left(\omega, \omega_{l}\right)=\left(\left\{\omega^{\prime}: \omega_{\mathscr{R}} \omega\right\} \cup\left\{S^{n}\left(\omega_{1}, \omega_{1}\right): n \geq 0\right\}\right)$ $\mathbf{t}_{l}\left(\omega, \omega_{l}\right)=\left(\left\{\omega_{i}^{i} \omega_{i}^{\prime} \mathscr{R}_{l}^{0} \omega_{l}\right\} \cup\left\{S_{l}^{n}\left(\omega_{1}, \omega_{l}\right): n \geq 0\right\}\right)$

(d) $\mathscr{T}=\left\{\mathbf{t}\left(w_{,} \omega_{l}\right): w \in \mathscr{W}\right.$ and $\left.w_{1} \in w_{.2}\right\}$

(e) lin $\left[w_{1}, t\left(w_{1}, w_{l}\right)\right]$ is the relation between $t\left(\omega, w_{l}\right)$ and $t_{l}\left(w, w_{l}\right)$ defined as follows:

if $\omega^{\prime} R \omega$, then $\left\langle\omega^{\prime}, \omega_{i}^{\prime}\right\rangle \in \operatorname{lin}\left[\omega_{,} w_{1}, t\left(w_{1}, w_{l}\right)\right]$ iff $\omega_{i}^{\prime} \in \omega^{\prime} .2$ and $\omega_{i}^{\prime} \mathscr{R}_{i}^{0} w_{l}$ for every $n \geq 0,\left\langle S^{n}\left(\omega, \omega_{i}\right), \omega_{i}^{\prime}\right\rangle \in \operatorname{lin}\left[\omega_{1}, \omega_{i}, t\left(\omega_{1}, \omega_{i}\right)\right]$ iff $\omega_{i}^{\prime}=S_{l}^{n}\left(\omega_{,} \omega_{i}\right)$

It is easy to see that the set of trajectories $\mathscr{T}$ defined in (d) satisfies the conditions imposed on the interpretation structures for $\mathscr{L}$. Moreover, using the next result, we also can prove that all the conditions described in (II) and (III) can be fulfilled.

\section{Proposition:}

Suppose that $w \in W$ and $w_{l} \in w .2$ and define $\mathscr{M}_{l}\left(w_{l}\right)(p)=1$ iff $p \in w_{l}$. then:

(a) lin $\left[w, w_{1}, t\left(w_{1}, w_{1}\right)\right]$ is an isomorphism between

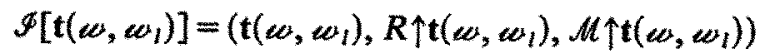

and

$$
\mathscr{I}_{l}=\left(\mathbf{t}_{l}\left(\omega, \omega_{l}\right), \mathscr{R}_{l} \uparrow \mathbf{t}\left(\omega, w_{l}\right), M_{i} \uparrow \mathbf{t}_{i}\left(\omega, \omega_{l}\right)\right),
$$


such that:

$$
\begin{aligned}
& \operatorname{lin}\left[\omega, \omega_{1}, t\left(\omega_{1} \omega_{l}\right)\right](\omega)=\left(\omega_{1}\right) \text {; } \\
& \operatorname{lin}\left[\omega, w_{1}, \mathbf{t}\left(w_{1} w_{l}\right)\right]\left(w^{\prime}\right) \in w^{\prime} .2 \text { for every } w^{\prime} \in \mathbf{t}\left(w_{1}, w_{l}\right) \text {. }
\end{aligned}
$$

(b) If $w_{l}^{\prime} \in \mathbf{t}_{l}\left(w_{1} w_{l}\right),\left(H f_{l}\right) \in L s u b f(\mathbf{f})$ and $\neg\left(H_{f_{l}}\right) \in \omega_{l}^{\prime}$, then there exists $w_{l}^{\prime \prime} \in$ $t_{l}\left(w, w_{l}\right)$ such that $w_{1}^{\prime \prime} \mathscr{R}_{1} w_{1}^{\prime}$ and $\neg f_{1} \in w_{l}^{\prime \prime}$.

(c) If $\omega_{l}^{\prime} \in \mathbf{t}_{l}\left(\omega_{1}, w_{1}\right),\left(G f_{l}\right) \in \operatorname{Lsubf}(\mathbf{f})$ and $\neg\left(G f_{1}\right) \in w_{l}^{\prime}$, then there exists $w_{l}^{\prime \prime} \in$ $t_{l}\left(w, w_{l}\right)$ such that $w_{l}^{\prime} \mathscr{R}_{l} w_{l}^{\prime \prime}$ and $\neg f_{l} \in w_{l}^{\prime \prime}$.

Finally, note that this completeness proof did not assume the completeness of the underlying infinitary linear axiomatisation. However, we can derive its completeness from the completeness of the infinitary axiomatisation for $\mathscr{L}$, taking into account the relationships between these axiomatisations, we well as does between the underlying semantics. On the other hand, for the infinitary linear axiomatisation we can also prove directly the following stronger result: "any consistent, set is satisfiable".

\section{Concluding Remarks}

An overview of the temporal formalisms used in concurrent program verification and in database and information systems specification was presented. The properties of the underlying time structures considered in the two areas were discussed. The branching versus path formulas controversy was revisited and the "for all trajectories" operator considered in CTL* [EmH86] was compared with the meta-linear operator $L$ proposed in [Car85].

The semantic properties of the $L$ operator as well as the semantic relationships with the underlying linear and pure branching logics were briefly analysed. This operator was introduced as a frame change operator and other interesting operators of this kind were illustrated.

The comparative advantages of the linear and branching approaches were analysed, in general and within the context of a layered methodology for systems specification. The usefulness of the operator $L$ for defining a unifying logic framework supporting such a layered methodology was illustrated.

Finitary and infinitary sound axiomatisations were proposed for the operator $L$ and for the underlying linear and pure branching logics. Their specific properties and the relationships between them were also referred. Finally, the proof of the completeness of the infinitary axiomatisation proposed for the operator $L$ was outlined. For this proof it was necessary to extend the usual Henkin constructions based on the maximal consistent sets in an appropriate way, in virtue of the frame change character of $L$.

The analysis of the possible interest of the operator $L$ in other applications seems to deserve further attention. More generally, we believe that this and other frame change operators will be useful in many applications, even if they are not yet in (informal) use.

A more technical direction for future research is proving the completeness of the finitary axiomatisation proposed for the operator $L$, using e.g. the techniques herein considered for the infinitary axiomatisation. Besides that, the independence, or not, of axioms (L10) and (L11), within that finitary axiomatisation, also needs further research.

Finally, no decidability results have been discussed herein. That is yet another obvious direction for future research. 


\section{Acknowledgments}

The authors wish to thank J. Fiadeiro and C. Sernadas for many fruitful discussions on some aspects of this paper. The authors also wish to thank the anonymous referee for his suggestions and criticisms that helped improve the readability and effectiveness of the paper.

\section{References}

[All83] Allen, J. F.: Maintaining Temporal Knowledge about Temporal Intervals. Comm. ACM, 26 (11) (1983).

[BMP81] Ben-Ari, M., Manna, Z. and Pnueli, A.: The Temporal Logic of Branching Time. Proc. 8th Annual Symposium on Principles of Programming Languages, ACM, pp. 164-175, 1981.

[Ben82] Benthem, J. F. A. K. van: The Logic of Time, Dordrecht: D. Reidel Publishing Company, 1982.

[Boo79] Boolos, G.: The Unprovability of Consistency. An Essay in Modal Logic, Cambridge University Press, 1979.

[Bub77] Bubenko, J.: The Temporal Dimension in Information Processing. In: Architecture and Models in Database Management, G. Nijssen, (ed.), North-Holland, 1977.

[Bur78] Burgess, J. P.: The Unreal Future. Theoria, XLIV, pp. 157-174 (1978).

[Car85] Carmo, J.: The Infolog Branching Logic of Events. In: Theoretical and Formal Aspects of Information Systems 1985, A. Sernadas, J. Bubenko, and A. Olivé (eds), NorthHolland, pp. 159-174, 1985.

[Car88] Carmo, J.: Lógicas Temporais para a Especificação e Verificação de Sistemas de Informação, Ph.D. thesis, IST (the Lisbon Institute of Technology, Technical University of Lisbon), 1988.

[CaS88a] Carmo, J. and Sernadas, A.: A Temporal Logic Framework for a Layered Approach to Systems Specification and Verification. In: Temporal Aspects in Information Systems, C. Rolland, F. Bodard and M. Leonard (eds), North-Holland, pp. 31-46, 1988.

[CaS88b] Carmo, J. and Sernadas, A.: Formal Techniques for Systems Specification and Verification, internal report, IST/INESC, Lisbon, 1988.

[CaS89a] Carmo, J. and Sernadas, A.: Inevitability in Branching Time. In: Logic at Botik '89, A. R. Mayer and M. A. Taitslin (eds), LNCS 363 Springer-Verlag, pp. 41-62, 1989.

[CaS89b] Carmo, J. and Sernadas, A.: A Completeness Result in a Mixed Branching-Linear Logic, to appear in the proceedings of the Logic Colloquium in Memory of Hugo Ribeiro (Lisbon), 1989.

[CaF82] Casanova, M. A. and Furtado, A. L.: On the Description of Database Transition Constraints Using Temporal Languages. Proc. 2nd Workshop for Logical Basis for Data Bases, pp. 211-236, 1982.

[CCF82] Castilho, J. M. V., Casanova, M. A. and Furtado, A. L.: A Temporal Framework for Information Systems Specification. Proc. 8th VLDB Conference, pp. 280-291, 1982.

[Che80] Chellas, B. F.: Modal Logic: An Introduction, Cambridge University Press, 1980.

[CIE81] Clarke, E. M. and Emerson, E. A.: Design and Synthesis of Synchronization Skeletons Using Branching Time Temporal Logic. Proc. Workshop on Logic of Programs, LNCS 131, Springer-Verlag, pp. 52-71, 1981.

[CGK89] Clarke, E. M., Grumberg. O. and Kurshan, R. P.: A Synthesis of Two Approaches for Verifying Finite State Concurrent Systems. In: Logic at Botik '89, A. R. Mayer and M. A. Taitslin (eds), LNCS 363, Springer-Verlag, pp. 81-90, 1989.

[Dij75] Dijkstra, E. W.: Guarded Commands, Nondeterminacy and Formal Derivation of Programs. Comm. ACM, 18 (8), 453-457 (1975).

[ESS88] Ehrich, H.-D., Sernadas, A. and Sernadas, C.: Abstract Object Types for Databases. In: Advances in Object-Oriented Database Systems, Dittrich (ed.), Springer-Verlag, pp. 144-149, 1988.

[Eme83] Emerson, E.A.: Alternative Semantics for Temporal Logics. Theor. Comput. Sci. 26, 121-130 (1983).

[EmH82] Emerson, E.A. and Halpern, J.Y.: Decision Procedures and Expressiveness in the Temporal Logic of Branching Time. Proc. 14th Annual ACM Symposium on Theory of Computing, ACM, pp. 169-180, 1982 (first version) and Journal of Comput. and Syst. Sciences, 30, 1-24 (1985) (second version). 
[EmH86] Emerson, E. A. and Halpeern, J. Y.: "Sometimes" and "Not Never" Revisited: On Branching versus Linear Time Temporal Logic. Journal ACM, 33 (1), 151-178 (1986).

[End72] Enderton, B.: A Mathematical Introduction to Logic, Academic Press, 1972.

[Fia89] Fiadeiro, J.: Cálculo de Objectos e Eventos, Ph.D. Thesis, IST (the Lisbon Institute of Technology, Technical University of Lisbon), 1989.

[FiS86] Fiadeiro, J. and Sernadas, A.: The Infolog Linear Tense Propositional Logic of Events and Transactions. Information Systems, 11 (1), 61-85 (1986).

[FiS88] Fiadeiro, $J$. and Sernadas, A.: Specification and Verification of Database Dynamics. Acta Informatica, 25, 625-661 (1988).

[FiL79] Fischer, M. and Ladner, R.: Propositional Dynamic Logic of Regular Programs. Journal of Comput. and Syst. Sciences, 18, 194-211 (1979).

[FuN86] Furtado, A. L. and Neuhold, E. J.: Formal Technics for Data Base Design, SpringerVerlag, 1986.

[Gab72] Gabbay, D. M.: Tense Systems with Discrete Moments of Time: Part I. Journal of Philosophical Logic, 1, 35-44 (1972).

[GPS80] Gabbay, D. M., Pnueli, A., Shelah, S. and Stavi, J.: On the Temporal Analysis of Fairness. Proc. 7th Anmual ACM Symposium on Principles of Programming Languages, ACM, pp. 163-173, 1980.

[Gol82] Goldblatt, R.: Axiomatizing the Logic of Computer Programming, LNCS 137, SpringerVerlag, 1982.

[GMS83] Golshani, F., Maibaum, T. and Sadler, M.: A Modal System of Algebras for Database Specification and Query/Update Language Support. Proc. 9th VLDB Conference, pp. $331-339,1983$.

[Har79] Harel, D.: First-Order Dynamic Logic, LNCS 68, Springer-Verlag, 1979.

[HKP82] Harel, D., Kozen, D. and Parikh, R.: Process Logic: Expressiveness, Decidability, Completeness. Journal of Comput and Syst. Sciences, 144-170 (1982).

[Hoa69] Hoare, C. A. R.: An Axiomatic Basis for Computer Programming. Comm. ACM, 12, 576-580, 1969.

[HuC68] Hughes, G. E. and Cresswell, M. J.: An Introduction to Modal Logic, Methuen and Co., 1968.

[HuC84] Hughes, G. E, and Cresswell, M. J.: A Companion to Modal Logic, Methuen and Co., 1984.

[Kam68] Kamp, H.: On Tense Logic and the Theory of Linear Order, Ph. D. thesis, University of California, Los Angeles, 1968.

[Kun84] Kung, C.: A Temporal Framework for Information Systems Specification and Verification, Ph. D. thesis, Norwegian Institute of Technology, 1984.

[Kun85] Kung, C.: A Tableaux Approach for Consistency Checking. In: Theoretical and Formal Aspects of Information Systems 1985, A. Sernadas, J. Bubenko and A. Olivé (eds), North-Holland, pp. 191-207, 1985.

[Lam80] Lamport, L.: "Sometime" is Sometimes "Not Never" on the Temporal Logic of Programs. Proc. 7th Annual ACM Symposium on Principles of Programming Languages, ACM, pp. 174-185, 1980.

[LeS82] Lehmann, D. and Shelah, S.: "Reasoning with Time and Chance. Information and Control, 53, 165-198 (1982).

[LPZ85] Lichtenstein, O., Pnueli, A. and Zuck, L.: The Glory of the Past. In: Logics of Programs, R. Parikh, (ed.), LNCS 193, Springer-Verlag, pp. 196-218, 1985.

[Lip88] Lipeck, U. W.: Transformation of Dynamic Integrity Constraints into Transaction Specifications. Proc. Int. Conf. on Database Theory 1988, LNCS, Springer-Verlag 1988.

[LEG85] Lipeck, U. W., Ehrich, H. D. and Gogolla, M.: Specifying Admissibility of Dynamic Database Behaviour Using Temporal Logic. In: Theoretical and Formal Aspects of Information Systems 1985, A. Sernadas, J. Bubenko and A. Olivé (eds), North-Holland, pp. $145-157,1985$.

[MKJ86] Maibaum, T. S. E., Khosla, S. and Jeremaes, P.: A Modal (Action) Logic for Requirements Specification. In: IEE Computing Series 6, D. Barnes and P. Brown (eds), Peter Peregrinus, 1986.

[MaP81] Manna, Z. and Pnueli, A.: Verification of Concurrent Programs: the Temporal Framework. In: The Correctness Problem in Computer Science, R. Boyer and J. Moore (eds), ILCS, Academic Press, pp. 215-273, 1981.

[MaP83] Manna, Z. and Pnueli, A.: Proving Precedence Properties: the Temporal Way. Automata Languages and Programming, 10th Colloquium, LNCS 154, Springer-Verlag, pp. 491$512,1983$.

[McA76] McArthur, R. P.: Tense Logic, D. Reidel Publishing Company, 1976. 
[McC79] McCall, S.: The Strong Future Tense. Notre Dame Journal of Formal Logic, XX (3), 489-504 (1979).

[NPW80] Nielsen, M., Plotkin, G. and Winskel, G.: Petri Nets, Event Structures, and Domains, Part 1. Theoretical Computer Science, 13 (1), 85-108 (1980).

[Nis80] Nishimura, H.: Descriptively Complete Process Logic. Acta Informatica, 14 (4), 359-369 (1980).

[OwL82] Owicki, S. and Lamport, L.: Proving Liveness Properties of Concurrent Programs. ACM TOPLAS, 4 (3), 455-495 (1982).

[Pnu77] Pnueli, A.: The Temporal Logic of Programs. Proc. 18th Annual ACM Symposium on Foundations of Computer Science, IEEE, pp. 45-57, 1977.

[Pnu79] Pnueli, A.: The Temporal Semantics of Concurrent Programs. In: Semantics of Concurrent Computation, G. Kahn (ed.), LNCS 70, Springer-Verlag, pp. 1-20, 1979.

[Pnu86] Pnueli, A.: Specification and Development of Reactive Systems. In: Information Processing 86, H. J. Kugler, (ed.), pp. 845-858, IFIP, 1986.

[Pra76] Pratt, V. R.: Semantical Considerations on Floyd-Hoare Logic. Proc. 17th Annual IEEE Symposium on Foundations of Computer Science, pp. 109-121, 1976.

[Pra87] Pratt, V. R.: Modelling Concurrency with Partial Orders. International Journal of Parallel Programming, 15 (1), 33-71 (1987).

[Pri67] Prior, A.: Past, Present and Future, Oxford, 1967.

[Rei88] Reisig, W.: Concurrency is More Fundamental than Interleaving. EATCS Bulletin, 35, 181-185 (1988).

[ReU71] Rescher, N. and Urquhart, A.: Temporal Logic, Springer-Verlag, 1971.

[RLR79] Rolland, C., Leifert, S. and Richard, C.: Tools for Information Systems Dynamics Management. Proc. Sth VLDB Conference, 1979.

[Sch85] Schiel, U: The Time Dimension in Information Systems. In: Theoretical and Formal Aspects of Information Systems, A. Sernadas, J. Bubenko and A. Olivé (eds), NorthHolland, pp. $67-75,1985$.

[Ser80] Sernadas, A.: Temporal Aspects of Logical Procedure Definition. Information Systems, $5(2), 167-187$ (1980).

[SeS83] Sernadas, A. and Sernadas, C.: Infolog: an Integrated Model of Data and Processes, INFOLOG RR05, IFIP WG 8.1 Meeting, York, 1983.

[SeS85] Sernadas, A. and Sernadas, C.: Capturing Knowledge About the Organization Dynamics. In: Knowledge Representation for DDS, L. B. Methlie and R. Sprague (eds), North-Holland, 1985

[SCS82] Sernadas, A., Carmo, J. and Sernadas, C.: Software Behavior Specification with Triggering Logic, INFOLOG RR02, Faculty of Sciences, Lisbon, 1982.

[SEC89] Sernadas, A., Ehrich, H.-D. and Costa, J.-F.: From Processes to Objects, internal report, IST/INESC, Lisbon, 1989.

[SSE87] Sernadas, A., Sernadas, C. and Ehrich, H.-D.: Object-Oriented Specification of Databases: An Algebraic Approach. In: Proc. 13th Conference on Very Large Data Bases, P. Hammersley (ed.), 1987.

[SFS88] Sernadas, A., Fiadeiro, J., Sernadas, C. and Ehrich, H.-D.: Abstract Object Types: A Temporal Perspective. In: Colloquium on Temporal Logic and Specification, A. Pnueli, H. Barringer and B. Baniegbal (eds), Springer-Verlag (to be published).

[Sti87] Stirling, C.: Comparing Linear and Branching Time Temporal Logics, to appear in Proc. Alvey Colloquium on Temporal Logics, Springer-Verlag, 1987.

[Tho70] Thomason, R. H.: Indeterministic Time and Truth-Value Gaps. Theoria, 36, 264-281 (1970).

[VeF85] Veloso, P. and Furtado, A.L.: Towards Simpler and Yet Complete Formal Specifications. In: Theoretical and Formal Aspects of Information Systems 1985, A. Sernadas, J. Bubenko and A. Olivé (eds), North-Holland, pp. 175-189, 1985.

[Ven88] Venema, Y. de: Expressiveness and Completeness of an Interval Tense Logic, ITLI Prepublication Series 88-02, Institute for Language, Logic and Information, University of Amsterdam, 1988.

[Zan89] Zanardo, A.: A Complete Deductive System for Since-Until Branching-Time Logic, internal report, Dipartimento di Matematica Pura ed Applicata, Universita' Degli Studi di Padova, 1989.

Received August 1988

Accepted in a revised form in November 1989 by C. B. Jones 